COMMUNICATIONS IN

ANALYSIS AND GEOMETRY

Volume 14, Number 5, 1037-1077, 2006

\title{
Heegaard genus of the connected sum of m-small knots
}

\author{
Tsuyoshi Kobayashi And Yo’AV Rieck
}

\begin{abstract}
We prove that if $K_{1} \subset M_{1}, \ldots, K_{n} \subset M_{n}$ are m-small knots in closed orientable 3 -manifolds, then the Heegaard genus of $E\left(\#_{i=1}^{n} K_{i}\right)$ is strictly less than the sum of the Heegaard genera of the $E\left(K_{i}\right)(i=1, \ldots, n)$ if and only if there exists a proper subset $I$ of $\{1, \ldots, n\}$ so that $\#_{i \in I} K_{i}$ admits a primitive meridian. This generalizes the main result of Morimoto [On the super additivity of tunnel number of knots, Math. Ann. 317 (2000), 489-508].
\end{abstract}

\section{Introduction}

Let $K_{i}(i=1, \ldots, n)$ be knots in closed orientable 3 -manifolds $M_{i}$, and let $K\left(=\#_{i=1}^{n} K_{i}\right) \subset M\left(=\#_{i=1}^{n} M_{i}\right)$ be their connected sum. Let $X=E(K)$ be the exterior of $K$ and $X_{i}$ be the exteriors of $K_{i}$. For $n=2$, it is well known that $g(X) \leq g\left(X_{1}\right)+g\left(X_{2}\right)$, and by induction, we see that for any $n, g(X) \leq \sum_{i=1}^{n} g\left(X_{i}\right)$, where $g(\cdot)$ denotes the Heegaard genus (definitions are given in Section 2). In this paper, we study the phenomenon $g(X)<$ $\sum_{i=1}^{n} g\left(X_{i}\right)$. We note that (for $n=2$ ) the phenomena $g(X)=g\left(X_{1}\right)+g\left(X_{2}\right)$ and $g(X) \leq g\left(X_{1}\right)+g\left(X_{2}\right)-k$ (for arbitrary $k$ ) can both occur (see [13, 18] for the former, $[15,7]$ for the latter). An instrumental definition for our work is given in [12, Definition 4.6].

Definition 1.1. A knot exterior $X$ admits a primitive meridian if there exists a minimal genus Heegaard surface $\Sigma \subset X$ that separates $X$ into a compression body $C$ and a handlebody $V$ so that there exist a compressing disk $D \subset V$ and a vertical annulus $A \subset C$ with $A \cap \partial X$ a meridian, and $\partial D \subset \Sigma$ intersects $A \cap \Sigma$ transversely in one point. (For the definition of vertical annulus, see Remarks 2.2(5).)

For knots admitting primitive meridians, we have: ${ }^{1}$

\footnotetext{
${ }^{1}$ Proposition 1.3 of [16] is stated in terms of the 1-bridge genus. However, by [16, Proposition 2.1], it is equivalent to this proposition.
} 
Proposition 1.2 [16, Propositions 1.3 and 2.1]. Let $K_{1}, K_{2}$ be knots in closed orientable 3-manifolds. Let $X_{1}, X_{2}$ and $X$ be the exteriors of the knots $K_{1}, K_{2}$ and $K_{1} \# K_{2}$, respectively. If $K_{1}$ or $K_{2}$ admits a primitive meridian, then $g(X) \leq g\left(X_{1}\right)+g\left(X_{2}\right)-1$.

Thus, existence of primitive meridian is a sufficient condition for $g(X)<$ $g\left(X_{1}\right)+g\left(X_{2}\right)$. A knot is called meridionally small (or $m$-small) if there is no essential surface in $E(K)$ with non-empty boundary so that each boundary component is a meridian. In [16, Theorem 1.6], Morimoto showed that for m-small knots in $S^{3}$, existence of primitive meridian is also necessary for $g(X)<g\left(X_{1}\right)+g\left(X_{2}\right)$ and conjectured that this holds for any two knots in $S^{3}$.

Conjecture 1.3 (Morimoto's conjecture). Let $K_{1}, K_{2}$ be knots in $S^{3}$. Let $X_{1}, X_{2}$ and $X$ be the exteriors of $K_{1}, K_{2}$ and $K_{1} \# K_{2}$, respectively. Then, $g(X)<g\left(X_{1}\right)+g\left(X_{2}\right)$ if and only if $X_{1}$ or $X_{2}$ admits a primitive meridian.

In this paper, we prove the following generalization of [16, Theorem 1.6].

Theorem 1.4. Let $K_{1}, \ldots, K_{n}(n \geq 2)$ be $m$-small knots in closed orientable 3-manifolds. Let $X_{1}, \ldots, X_{n}$ and $X$ be the exteriors of $K_{1}, \ldots, K_{n}$ and $\#_{i=1}^{n} K_{i}$, respectively. Then, $g(X)<\sum_{i=1}^{n} g\left(X_{i}\right)$ if and only if there exists $I \neq \emptyset$ a proper subset of $\{1, \ldots, n\}$ such that the exterior of $\#_{i \in I} K_{i}$ admits a primitive meridian.

\section{Remarks 1.5.}

(1) One might hope for the following simple statement as the conclusion of Theorem 1.4: given knots $K_{1}, \ldots, K_{n}, g(X)<\Sigma_{i=1}^{n} g\left(X_{i}\right)$ holds if and only if for some $i, K_{i}$ admits a primitive meridian. However, the following examples show that this is not the case. Let $K_{m}(m \in \mathbb{Z})$ be the knots introduced by Morimoto et al. in [18]. It is shown in [18] that for each $m, g\left(E\left(K_{m}\right)\right)=2, K_{m}$ does not admit a primitive meridian, and $g\left(E\left(K_{m} \# K_{m}\right)\right)=4$. On the other hand, in [10, Section $5]$, it is shown that $E\left(K_{m} \# K_{m}\right)$ admits a primitive meridian. Hence, by Proposition 1.2, $g\left(E\left(K_{m} \# K_{m} \# K_{m}\right)\right)<4+2=3 g\left(E\left(K_{m}\right)\right)$.

(2) Many of the ideas used for the proof of Theorem 1.4 can be found in [17] where Morimoto proved [17, Theorem 1.1] that if $K_{i}(i=1, \ldots, n)$ are m-small knots in closed orientable 3 -manifolds $M_{i}$ so that no $M_{i}$ contains a lens space summand, then $g(X) \geq \sum_{i=1}^{n} g\left(X_{i}\right)-(n-1)$. In this 
paper, we retrieve this result (Corollary 5.3) without the restriction on the summands of the manifolds $M_{i}$.

The "if" part of Theorem 1.4 is an easy consequence of Proposition 1.2. We now sketch the proof of the "only if" part and introduce the main tools applied. The proof is an induction on the number of summands $n$. Our first tool, the Swallow Follow Torus Theorem (Theorem 4.1), implies that if $K_{1}, \ldots, K_{n}(n \geq 2)$ are $\mathrm{m}$-small knots, then any Heegaard surface for $X\left(=E\left(\#_{i=1}^{n} K_{i}\right)\right)$ weakly reduces to a swallow follow torus (say $T$ ). In particular, $X$ does not admit a strongly irreducible Heegaard splitting. It follows from the definition of swallow follow torus that $T$ decomposes $X$ as $X=X_{I}^{(1)} \cup_{T} X_{J}$, with $I \subset\{1, \ldots, n\}$ a non-empty proper subset, $X_{I}=$ $E\left(\#_{i \in I} K_{i}\right), X_{J}=E\left(\#_{i \notin I} K_{i}\right)$ and $X_{I}^{(1)}$ is obtained from $X_{I}$ by drilling out a simple closed curve parallel to the meridian of $K_{I}$. If $g(X)=g\left(X_{I}\right)+g\left(X_{J}\right)$, then either $g\left(X_{I}\right)<\Sigma_{i \in I} g\left(E\left(K_{i}\right)\right)$ or $g\left(X_{J}\right)<\Sigma_{i \in J} g\left(E\left(K_{i}\right)\right)$ (say the former). By induction, Theorem 1.4 holds for $X_{I}$, and hence for some $I^{\prime} \subset I$, $E\left(\#_{i \in I^{\prime}} K_{i}\right)$ admits a primitive meridian, and we are done. Thus, we may assume that $g(X)<g\left(X_{I}\right)+g\left(X_{J}\right)$. In that case, Corollary 5.4 implies that $g\left(X_{I}^{(1)}\right)=g\left(X_{I}\right)$.

Our second tool, the Hopf-Haken annulus theorem (Theorem 6.3), studies minimal genus Heegaard splitting of $X_{I}^{(1)}$. Denote the boundary of $X_{I}^{(1)}$ by $\partial X_{I} \cup T$. Note that $X_{I}^{(1)}$ admits an essential annulus (say $A$ ) connecting $\partial X_{I}$ to $T$, so that $A \cap \partial X_{I}$ is a meridian of $X_{I}$ and $A \cap T$ is a longitude of $T$. The Hopf-Haken annulus theorem implies that if $K_{I}=\#_{i \in I} K_{i}$ is the connected sum of m-small knots, then there exists a minimal genus Heegaard surface for $X_{I}^{(1)}$ (say $\Sigma$ ) that intersects $A$ in a single simple closed curve that is essential in both $A$ and the Heegaard surface. Applying this to

$X_{I}^{(1)}$ described in the previous paragraph, since $g\left(X_{I}\right)=g\left(X_{I}^{(1)}\right)$, we have that $\Sigma$ is also a minimal genus Heegaard surface for $X_{I}$. It is then easy to see (Proposition 6.2) that $\Sigma$ fulfills the conditions of Definition 1.1 and hence $X_{I}$ admits a primitive meridian, proving Theorem 1.4. Thus, the Hopf-Haken annulus theorem connects the Swallow Follow Torus Theorem to the existence of a primitive meridian.

\section{Background}

Throughout this paper, we work in the category of smooth manifolds. All manifolds considered are assumed to be orientable. For a submanifold $H$ of a manifold $M, N(H, M)$ denotes a regular neighborhood of $H$ in $M$. When 
$M$ is understood from context, we often abbreviate $N(H, M)$ to $N(H)$. Let $F$ be a surface. Then a surface in $F \times[0,1]$ is called vertical if it is ambient isotopic to a surface of the form $c \times[0,1], c \subset F$ an embedded 1-manifold. Let $N$ be a manifold embedded in a manifold $M$ with $\operatorname{dim}(N)=\operatorname{dim}(M)$. Then, the frontier of $N$ in $M$ is denoted by $\operatorname{Fr}_{M}(N)$. For definitions of standard terms of 3 -dimensional topology, we refer to $[5,6]$.

The following lemma is a well-known fact. Informally, it says that for manifolds with torus boundary compression implies compression; for a proof see, for example, [9, Lemma 2.7].

Lemma 2.1. Let $N$ be a 3-manifold with a torus boundary component $T$. Let $S$ be a 2-sided surface properly embedded in $N$ such that $S \cap T$ consists of essential simple closed curves in T. Suppose that there is a boundary compressing disk $\Delta$ for $S$ such that $\Delta$ compresses $S$ into $T$, i.e., $\Delta \cap \partial N=$ $\partial \Delta \cap T$ is an arc, say $\alpha$, and $\Delta \cap S=\partial \Delta \cap S$ is an essential arc in $S$, say $\beta$, such that $\alpha \cup \beta=\partial \Delta$. Then, we have either one of the following.

(1) $S$ is compressible. Moreover, if $S$ is separating in $N$, then the compression occurs in the same side as the boundary compression.

(2) $S$ is an annulus; moreover, when $N$ is irreducible, $S$ is boundary parallel.

Let $S$ be a surface properly embedded in a 3 -manifold $N$. Then $S$ is called essential if $S$ is incompressible and not boundary parallel. If $N$ is irreducible and $\partial N$ consists of tori, then by Lemma 2.1, $S$ being essential implies $S$ is not boundary compressible.

A 3-manifold $C$ is called a compression body if there is a compact, connected, closed, orientable surface $F$ such that $C$ is obtained from $F \times$ $[0,1]$ by attaching 2 -handles along mutually disjoint simple closed curves in $F \times\{1\}$ and capping off the 2 -sphere boundary components using 3 -handles. Then, $F \times\{0\}$ is denoted by $\partial_{+} C$ and $\partial_{-} C=\partial C \backslash \partial_{+} C$. A compression body is called a handlebody if $\partial_{-} C=\emptyset$. A compression body $C$ is called trivial if $C$ is homeomorphic to $F \times[0,1]$ with $\partial_{+} F=F \times\{0\}$ and $\partial_{-} F=$ $F \times\{1\}$. A disk $D$ embedded in a compression body $C$ is called a meridian disk if $\partial D$ is non-trivial in $\partial_{+} C$. (We note that $\partial_{-} C$ is incompressible.) By extending the cores of the 2-handles in the definition of a compression body vertically in $F \times[0,1]$, we obtain a union of mutually disjoint meridian disks in $C$, say $\mathcal{D}$, such that the manifold obtained from $C$ by cutting along $\mathcal{D}$ is homeomorphic to a union of $\partial_{-} C \times[0,1]$ and a (possibly empty) union of 3balls corresponding to the attached 3 -handles. This gives a dual description 
of compression bodies, that is, a connected 3-manifold $C$ is a compression body if there exists a closed (not necessarily connected) surface $F$ without 2 -sphere components and a (possibly empty) union of 3-balls (say $\mathcal{B}$ ) so that $C$ is obtained from $(F \times[0,1]) \cup \mathcal{B}$ by attaching 1-handles along $(F \times\{0\}) \cup$ $\partial \mathcal{B}$. We may regard each component of $\mathcal{B}$ as a 0 -handle. Hence, $C$ admits a handle decomposition $(F \times[0,1]) \cup 0$-handles $\cup 1$-handles. We note that $\partial_{-} C$ is the surface corresponding to $F \times\{1\}$.

Remarks 2.2. The following properties are known for compression bodies (the proofs are omitted or outlined below):

(1) Compression bodies are irreducible.

(2) Let $\mathcal{D}$ be a union of mutually disjoint meridian disks of a compression body $C$ and $C^{\prime}$ a component of the manifold obtained by cutting $C$ open along $\mathcal{D}$. Then, $C^{\prime}$ inherits a compression body structure from $C$, i.e., $C^{\prime}$ is a compression body such that $\partial_{-} C^{\prime}=\partial_{-} C \cap C^{\prime}$ and $\partial_{+} C^{\prime}=\left(C^{\prime} \cap \partial_{+} C\right) \cup \operatorname{Fr}_{C}\left(C^{\prime}\right)$.

(3) Let $S$ be an incompressible surface in $C$ such that $\partial S \neq \emptyset$ and $\partial S \subset$ $\partial_{+} C$. Suppose that $S$ is not a union of meridian disks. Then $S$ is boundary compressible into $\partial_{+} C$ in $C$, i.e., there exists a disk $\Delta$ in $C$ such that $\Delta \cap S=\partial \Delta \cap S=a$ is an essential arc in $S$ and $\Delta \cap \partial C=$ $\Delta \cap \partial_{+} C=\operatorname{cl}(\partial \Delta \backslash a)$.

(4) Let $A$ be a surface properly embedded in $C$ such that $\partial A \subset \partial_{+} C$, and each component of $A$ is an essential annulus. Then by boundary compressing an outermost component of $A$ (see (3) above), we obtain a meridian disk $D$. By a tiny isotopy, we may assume that $D \cap A=\emptyset$. This implies the following: let $A \subset C$ be as above. Then there is a meridian disk $D$ of $C$ such that $D \cap A=\emptyset$.

(5) Let $S$ be an incompressible, boundary incompressible surface with non-empty boundary properly embedded in $C$. Then each component of $S$ is either a meridian disk or an annulus $A$ such that one component of $\partial A$ is in $\partial_{+} C$ and the other in $\partial_{-} C$. (Such an incompressible annulus is called vertical.)

(6) Let $S$ be an incompressible (possibly closed) surface properly embedded in $C$ such that $S \cap \partial_{+} C=\emptyset$. Then each component of $S$ is parallel to a subsurface of $\partial_{-} C$.

Let $N$ be a 3 -manifold and $F_{1}, F_{2}$ a partition of $\partial N$ (possibly, $F_{1}=\emptyset$ or $F_{2}=\emptyset$ ). 
Definition 2.3. We say that a decomposition $C_{1} \cup_{\Sigma} C_{2}$ is a Heegaard splitting of $\left(N ; F_{1}, F_{2}\right)$ if the following conditions hold:

(1) $C_{i}(i=1,2)$ is a compression body;

(2) $C_{1} \cup C_{2}=N$;

(3) $C_{1} \cap C_{2}=\partial_{+} C_{1}=\partial_{+} C_{2}=\Sigma$; and

(4) $\partial_{-} C_{i}=F_{i}(i=1,2)$.

The surface $\Sigma$ is called a Heegaard surface of $\left(N ; F_{1}, F_{2}\right)$. If the boundary partition of $N$ is irrelevant, we omit condition (4) in Definition 2.3 and say that $\Sigma$ is a Heegaard surface of $N$. The genus of the Heegaard splitting is the genus of $\Sigma$, denoted by $g(\Sigma)$ or $g\left(C_{1} \cup_{\Sigma} C_{2}\right)$. The minimal genus of all Heegaard splittings of $\left(N ; F_{1}, F_{2}\right)$ is called the (Heegaard) genus of $\left(N ; F_{1}, F_{2}\right)$ and is denoted by $g\left(N ; F_{1}, F_{2}\right)$. The minimal genus of all Heegaard splittings of $N$ is called the (Heegaard) genus of $N$ and is denoted by $g(N)$.

\section{Definitions 2.4.}

(1) A Heegaard splitting $C_{1} \cup_{\Sigma} C_{2}$ is called reducible if there exist meridian disks $D_{1} \subset C_{1}$ and $D_{2} \subset C_{2}$ such that $\partial D_{1}=\partial D_{2}$. Otherwise, the Heegaard splitting is called irreducible.

(2) A Heegaard splitting $C_{1} \cup_{\Sigma} C_{2}$ is called weakly reducible if there exist meridian disks $D_{1} \subset C_{1}$ and $D_{2} \subset C_{2}$ such that $\partial D_{1} \cap \partial D_{2}=\emptyset$. Otherwise, the splitting is called strongly irreducible.

(3) A Heegaard splitting $C_{1} \cup_{\Sigma} C_{2}$ is called stabilized if there exist meridian disks $D_{1} \subset C_{1}$ and $D_{2} \subset C_{2}$ such that $\partial D_{1}$ intersects $\partial D_{2}$ transversely in one point. Otherwise the splitting is said to be non stabilized.

(4) A Heegaard splitting $C_{1} \cup_{\Sigma} C_{2}$ is called trivial if $C_{1}$ or $C_{2}$ is a trivial compression body.

Let $C_{1} \cup_{\Sigma} C_{2}$ be a Heegaard splitting of $\left(N ; F_{1}, F_{2}\right)$. Recall that $C_{1}$ was obtained from $\left(F_{1} \times[0,1]\right) \cup 0$-handles by attaching 1 -handles and that $C_{2}$ was obtained from $\partial_{+} C_{2} \times[0,1]$ by attaching 2 - and 3 -handles. Then, by using an isotopy which pushes $\partial_{+} C_{2} \times[0,1]$ out of $C_{2}$, we identify $\partial_{+} C_{2} \times$ $[0,1]$ with $N\left(\partial_{+} C_{1}, C_{1}\right)$. This identification, together with the above handles, gives the following handle decomposition of $N$ :

$$
N=\left(F_{1} \times[0,1]\right) \cup 0 \text {-handles } \cup 1 \text {-handles } \cup \text { 2-handles } \cup 3 \text {-handles. }
$$


We say that this handle decomposition is induced by $C_{1} \cup_{\Sigma} C_{2}$. Suppose that there exists a proper subset of the 0 - and 1-handles so that some subset of the 2- and 3-handles does not intersect the subset of 0- and 1-handles. Suppose further that we can rearrange the order of the handles non-trivially to obtain an increasing sequence of (not necessarily connected) submanifolds $N_{1}, \ldots, N_{n}$ of $N$ such that the following holds:

$$
\begin{aligned}
N_{0}= & \emptyset \\
N_{j}= & N_{j-1} \cup\left(F_{1}^{(j-1)} \times[0,1]\right) \cup 0 \text {-handles } \cup \text { 1-handles } \\
& \cup \text { 2-handles } \cup \text { 3-handles }(1 \leq j \leq n),
\end{aligned}
$$

where $\left\{F_{1}^{(0)}, \ldots, F_{1}^{(n-1)}\right\}$ is a partition of the components of $F_{1}$ (with $F_{1}^{(j)}$ possibly empty, $0 \leq j \leq n-1$ ) and the handles appearing above come from a handle decomposition of $N$ induced by $C_{1} \cup_{\Sigma} C_{2}$, where each collection of 0 - and 3-handles may be empty but the collections of 1- and 2-handles are never empty. Suppose further that this handle decomposition satisfies the following three conditions:

(1) $N_{1}$ is connected.

(2) At each stage $j(1 \leq j \leq n)$, let $\partial_{j}^{-}$denote the union of the components of $\partial N_{j-1}$ to which the 1 -handles are attached. Then, $\partial_{j}^{-} \cup\left(F_{1}^{(j-1)} \times\right.$ $[0,1]) \cup 0$-handles $\cup$ 1-handles $\cup$ 2-handles $\cup 3$-handles is connected, where these handles are the handles that appear in the description of $N_{j}$.

(3) No component of $\partial N_{j}$ is a 2 -sphere $(j=1, \ldots, n-1)$.

We note that if $C_{1} \cup_{\Sigma} C_{2}$ is irreducible, then condition (3) holds automatically (this is a consequence of Lemma 2.10 and Proposition 2.13 of this paper). Then, for each $j$, let $I_{j}=\partial_{j}^{-} \times[0,1]$ and $R_{j}=I_{j} \cup\left(F_{1}^{(j)} \times[0,1]\right) \cup$ 0-handles $\cup$ 1-handles $\cup$ 2-handles $\cup$ 3-handles (handles that appear in the description of $\left.N_{j}\right)$, where $\partial_{j}^{-}\left(\subset \partial N_{j-1}\right)$ is identified with $\partial_{j}^{-} \times\{0\}$ $\left(\subset I_{j}\right)$. By the above conditions, we see that this handle decomposition induces a Heegaard splitting, say $C_{1}^{(j)} \cup C_{2}^{(j)}$, of $R_{j}$.

It is clear that $N$ can be regarded as obtained from $R_{1}, \ldots, R_{n}$ by identifying their boundaries. Hence, we have obtained a decomposition of $N$ into submanifolds $R_{1}, \ldots, R_{n}$ and Heegaard splittings for those submanifolds as follows:

$$
\left(C_{1}^{(1)} \cup C_{2}^{(1)}\right) \cup\left(C_{1}^{(2)} \cup C_{2}^{(2)}\right) \cup \cdots \cup\left(C_{1}^{(n)} \cup C_{2}^{(n)}\right) .
$$


This decomposition is called an untelescoping of $C_{1} \cup_{\Sigma} C_{2}$, originally defined in a slightly different way by Scharlemann and Thompson in [24]. An untelescoping is called a Scharlemann-Thompson untelescoping (or S-T untelescoping) of the Heegaard splitting $C_{1} \cup_{\Sigma} C_{2}$ if for each $j, C_{1}^{(j)} \cup C_{2}^{(j)}$ is strongly irreducible.

Remark 2.5. It is well known that every irreducible Heegaard splitting of a 3-manifold $M$ with incompressible boundary (other than surface crossinterval) admits an S-T untelescoping such that each Heegaard splitting is non-trivial $[23,24]$. In this case, every component of $\partial R_{j}$ is incompressible in $M$. If, in addition, $C_{1} \cup_{\Sigma} C_{2}$ is minimal genus, then by [27], each component of $\partial R_{j} \backslash \partial M$ is an essential surface.

\subsection{Amalgamation of Heegaard splittings}

Let $M^{(1)}$ and $M^{(2)}$ be compact, orientable 3-manifolds with boundary, together with partitions of the boundary components:

$$
\begin{aligned}
& \partial M^{(1)}=F^{(1)} \cup F_{1}^{(1)} \cup F_{2}^{(1)}, \\
& \partial M^{(2)}=F^{(2)} \cup F_{1}^{(2)} \cup F_{2}^{(2)},
\end{aligned}
$$

where $F^{(1)}$ and $F^{(2)}$ are non-empty and mutually homeomorphic. Let $M$ be a manifold obtained from $M^{(1)}$ and $M^{(2)}$ by identifying $F^{(1)}$ and $F^{(2)}$. Then $F$ denotes the image of $F^{(1)}$ (= the image of $F^{(2)}$ ) in $M$.

Suppose that we are given Heegaard splittings

$$
C_{1}^{(1)} \cup C_{2}^{(1)} \quad \text { of }\left(M^{(1)} ; F_{1}^{(1)}, F^{(1)} \cup F_{2}^{(1)}\right)
$$

and

$$
C_{1}^{(2)} \cup C_{2}^{(2)} \quad \text { of }\left(M^{(2)} ; F^{(2)} \cup F_{1}^{(2)}, F_{2}^{(2)}\right)
$$

Recall that there are handle decompositions

$$
M^{(1)}=\left(F_{1}^{(1)} \times[0,1]\right) \cup \mathcal{H}_{0}^{(1)} \cup \mathcal{H}_{1}^{(1)} \cup \mathcal{H}_{2}^{(1)} \cup \mathcal{H}_{3}^{(1)},
$$

and

$$
M^{(2)}=\left(F^{(2)} \times[0,1]\right) \cup\left(F_{1}^{(2)} \times[0,1]\right) \cup \mathcal{H}_{0}^{(2)} \cup \mathcal{H}_{1}^{(2)} \cup \mathcal{H}_{2}^{(2)} \cup \mathcal{H}_{3}^{(2)},
$$


where the $\mathcal{H}_{i}^{(j)}$ denotes the handles of index $i$ induced by the Heegaard splitting $C_{1}^{(j)} \cup C_{2}^{(j)}$. Hence, we have

$$
\begin{aligned}
M= & \left(F_{1}^{(1)} \times[0,1]\right) \cup \mathcal{H}_{0}^{(1)} \cup \mathcal{H}_{1}^{(1)} \cup \mathcal{H}_{2}^{(1)} \cup \mathcal{H}_{3}^{(1)} \\
& \cup\left(F^{(2)} \times[0,1]\right) \cup\left(F_{1}^{(2)} \times[0,1]\right) \cup \mathcal{H}_{0}^{(2)} \cup \mathcal{H}_{1}^{(2)} \cup \mathcal{H}_{2}^{(2)} \cup \mathcal{H}_{3}^{(2)},
\end{aligned}
$$

Then, via ambient isotopy, we push $M^{(2)}$ slightly into $M^{(1)}$ so that $F^{(2)} \times$ $[0,1]$ is identified with $N\left(F^{(1)}, M^{(1)}\right)$. Hence, $F^{(1)}\left(\subset \partial M^{(1)}\right)$ is regarded as $F^{(2)} \times\{0\} \subset \operatorname{int} M^{(2)}$. (Note that in the handle decomposition $M^{(2)}=$ $\left(F^{(2)} \times[0,1]\right) \cup\left(F_{1}^{(2)} \times[0,1]\right) \cup \mathcal{H}_{0}^{(2)} \cup \mathcal{H}_{1}^{(2)} \cup \mathcal{H}_{2}^{(2)} \cup \mathcal{H}_{3}^{(2)}$, the handles of $\mathcal{H}_{1}^{(2)}$ are attached to $\left(F^{(2)} \times\{0\}\right) \cup\left(F_{1}^{(2)} \times\{0\}\right) \cup \partial \mathcal{H}_{0}^{(2)}$. $)$ Here, via isotopy, we may suppose that $\mathcal{H}_{1}^{(2)}$ is disjoint from $\mathcal{H}_{1}^{(1)} \cup \mathcal{H}_{2}^{(1)}$ and that $\mathcal{H}_{2}^{(2)}$ is disjoint from $\mathcal{H}_{2}^{(1)}$. This implies that we can change the order of the handles so that

$$
\begin{aligned}
M= & \left(F_{1}^{(1)} \times[0,1]\right) \cup\left(F_{1}^{(2)} \times[0,1]\right) \cup\left(\mathcal{H}_{0}^{(1)} \cup \mathcal{H}_{0}^{(2)}\right) \\
& \cup\left(\mathcal{H}_{1}^{(1)} \cup \mathcal{H}_{1}^{(2)}\right) \cup\left(\mathcal{H}_{2}^{(1)} \cup \mathcal{H}_{2}^{(2)}\right) \cup\left(\mathcal{H}_{3}^{(1)} \cup \mathcal{H}_{3}^{(2)}\right) .
\end{aligned}
$$

By using this handle decomposition, we can obtain a Heegaard splitting, say $C_{1} \cup C_{2}$, of $M$. We say that $C_{1} \cup C_{2}$ is obtained from $C_{1}^{(1)} \cup C_{2}^{(1)}$ and $C_{1}^{(2)} \cup C_{2}^{(2)}$ by an amalgamation or that $C_{1} \cup C_{2}$ is an amalgamation of $C_{1}^{(1)} \cup C_{2}^{(1)}$ and $C_{1}^{(2)} \cup C_{2}^{(2)}$. We note that this definition of amalgamation has different appearance than that in [25]. However, it is easy to see that they yield the same surface.

By the definition of untelescoping, we immediately have the following:

Proposition 2.6. Let $\left(C_{1}^{(1)} \cup C_{2}^{(1)}\right) \cup \cdots \cup\left(C_{1}^{(n)} \cup C_{2}^{(n)}\right)$ be an untelescoping of $C_{1} \cup C_{2}$. Then, $C_{1} \cup C_{2}$ is obtained from $C_{1}^{(1)} \cup C_{2}^{(1)}, \ldots, C_{1}^{(n)} \cup C_{2}^{(n)}$ by a sequence of amalgamations.

The proof of Lemma 2.7 (e.g., Remark 2.7 of [25] for the case $m=1$ ) is elementary and is left to the reader.

Lemma 2.7. Let $C_{1} \cup C_{2}, C_{1}^{(1)} \cup C_{2}^{(1)}$ and $C_{1}^{(2)} \cup C_{2}^{(2)}$ be as above. Let $F_{1}, \ldots, F_{m}$ be the components of $F \subset M$. Then,

$$
g\left(C_{1} \cup C_{2}\right)=g\left(C_{1}^{(1)} \cup C_{2}^{(1)}\right)+g\left(C_{1}^{(2)} \cup C_{2}^{(2)}\right)-\sum_{i=1}^{m} g\left(F_{i}\right)+(m-1) .
$$


Proposition 2.8. Let $M$ be a compact 3-manifold with partition of the boundary components: $\quad \partial M=\left(F_{1}^{(1)} \cup F_{1}^{(2)}\right) \cup\left(F_{2}^{(1)} \cup F_{2}^{(2)}\right)$. Suppose that there exists a minimal genus Heegaard splitting $C_{1} \cup C_{2}$ of $\left(M ; F_{1}^{(1)} \cup F_{1}^{(2)}\right.$, $\left.F_{2}^{(1)} \cup F_{2}^{(2)}\right)$ such that $C_{1} \cup C_{2}$ admits an untelescoping

$$
\left(C_{1}^{(1)} \cup C_{2}^{(1)}\right) \cup_{F}\left(C_{1}^{(2)} \cup C_{2}^{(2)}\right)
$$

with the following properties:

- $\partial_{-} C_{1}^{(1)}=F_{1}^{(1)}$ and $\partial_{-} C_{2}^{(2)}=F_{2}^{(2)}$, and

- $\partial_{-} C_{2}^{(1)}=F^{(1)} \cup F_{2}^{(1)}$ and $\partial_{-} C_{1}^{(2)}=F^{(2)} \cup F_{1}^{(2)}$,

where $F^{(1)}$ and $F^{(2)}$ are the surfaces that are identified in $M$ as $F$. Then, $C_{1}^{(1)} \cup C_{2}^{(1)}$ and $C_{1}^{(2)} \cup C_{2}^{(2)}$ are minimal genus Heegaard splittings of $\left(M^{(1)} ; F_{1}^{(1)}, F^{(1)} \cup F_{2}^{(1)}\right)$ and $\left(M^{(2)} ; F^{(2)} \cup F_{1}^{(2)}, F_{2}^{(2)}\right)$, respectively. In particular, we have the following identity:

$$
\begin{aligned}
g\left(M ; F_{1}^{(1)} \cup F_{1}^{(2)}, F_{2}^{(1)} \cup F_{2}^{(2)}\right)= & g\left(M^{(1)} ; F_{1}^{(1)}, F_{2}^{(1)} \cup F^{(1)}\right) \\
& +g\left(M^{(2)} ; F_{1}^{(2)} \cup F^{(2)}, F_{2}^{(2)}\right) \\
& -\sum_{i=1}^{m} g\left(F_{i}\right)+(m-1),
\end{aligned}
$$

where $F_{1}, \ldots, F_{m}$ are the components of $F$.

Proof. If either $C_{1}^{(1)} \cup C_{2}^{(1)}$ or $C_{1}^{(2)} \cup C_{2}^{(2)}$ is not a minimal genus Heegaard splitting of $\left(M^{(1)} ; F_{1}^{(1)}, F_{2}^{(1)} \cup F^{(1)}\right)$ or $\left(M^{(2)} ; F_{1}^{(2)} \cup F^{(2)}, F_{2}^{(2)}\right)$, respectively, then by amalgamating minimal genus Heegaard splittings of $\left(M^{(1)} ; F_{1}^{(1)}\right.$, $F^{(1)} \cup F_{2}^{(1)}$ ) and $\left(M^{(2)} ; F_{1}^{(2)} \cup F^{(2)}, F_{2}^{(2)}\right)$, we see, by Lemma 2.7 , that we obtain a Heegaard splitting of $\left(M ; F_{1}^{(1)} \cup F_{1}^{(2)}, F_{2}^{(1)} \cup F_{2}^{(2)}\right)$ with genus lower than $g\left(C_{1} \cup C_{2}\right)$, contradicting the fact that the $C_{1} \cup C_{2}$ is a minimal genus Heegaard splitting of $\left(M ; F_{1}^{(1)} \cup F_{1}^{(2)}, F_{2}^{(1)} \cup F_{2}^{(2)}\right)$. Hence, $C_{1}^{(1)} \cup$ $C_{2}^{(1)}$ and $C_{1}^{(2)} \cup C_{2}^{(2)}$ are minimal genus Heegaard splittings of $\left(M^{(1)} ; F_{1}^{(1)}\right.$, $\left.F^{(1)} \cup F_{2}^{(1)}\right)$ and $\left(M^{(2)} ; F_{1}^{(2)} \cup F^{(2)}, F_{2}^{(2)}\right)$, respectively. This together with Lemma 2.7 implies

$$
\begin{aligned}
g\left(M ; F_{1}^{(1)} \cup F_{1}^{(2)}, F_{2}^{(1)} \cup F_{2}^{(2)}\right)= & g\left(M^{(1)} ; F_{1}^{(1)}, F_{2}^{(1)} \cup F^{(1)}\right) \\
& +g\left(M^{(2)} ; F_{1}^{(2)} \cup F^{(2)}, F_{2}^{(2)}\right) \\
& -\sum_{i=1}^{m} g\left(F_{i}\right)+(m-1) .
\end{aligned}
$$


Recall that $g(M)$ denotes the genus of the minimal genus Heegaard splitting of $M$ for all possible partitions of the components of $\partial M$.

Proposition 2.9. Let $N$ be a compact orientable 3-manifold with a minimal genus Heegaard splitting $C_{1} \cup_{\Sigma} C_{2}$ admitting an untelescoping $\left(C_{1}^{(1)} \cup C_{2}^{(1)}\right)$ $\cup_{F}\left(C_{1}^{(2)} \cup C_{2}^{(2)}\right)$, where $F$ is a connected surface. Let $M^{(1)}=C_{1}^{(1)} \cup$ $C_{2}^{(1)}$ and $M^{(2)}=C_{1}^{(2)} \cup C_{2}^{(2)}$. Then, $C_{1}^{(1)} \cup C_{2}^{(1)}$ and $C_{1}^{(2)} \cup C_{2}^{(2)}$ are minimal genus Heegaard splittings of $M^{(1)}$ and $M^{(2)}$, respectively. In particular, we have the following equality:

$$
g(M)=g\left(M^{(1)}\right)+g\left(M^{(2)}\right)-g(F) .
$$

Proof. Suppose that either $C_{1}^{(1)} \cup C_{2}^{(1)}$ or $C_{1}^{(2)} \cup C_{2}^{(2)}$, say $C_{1}^{(1)} \cup C_{2}^{(1)}$, is not a minimal genus Heegaard splitting. Let $\bar{C}_{1}^{(1)} \cup \bar{C}_{2}^{(1)}$ be a minimal genus Heegaard splitting of $M^{(1)}$. Since $F$ is connected, by exchanging $\bar{C}_{1}^{(1)}$ and $\bar{C}_{2}^{(1)}$ if necessary, we may assume that $F \subset \partial_{-} \bar{C}_{2}^{(1)}$. Hence, we can amalgamate $\bar{C}_{1}^{(1)} \cup \bar{C}_{2}^{(1)}$ and $C_{1}^{(2)} \cup C_{2}^{(2)}$ to obtain a Heegaard splitting, say $\bar{C}_{1} \cup_{\bar{\Sigma}} \bar{C}_{2}$ of $M$. Here we note that $g(\bar{\Sigma})<g(\Sigma)$ by Lemma 2.7 , a contradiction. Hence, $C_{1}^{(1)} \cup C_{2}^{(1)}$ and $C_{1}^{(2)} \cup C_{2}^{(2)}$ are minimal genus Heegaard splittings. This together with Lemma 2.7 implies

$$
g(M)=g\left(M^{(1)}\right)+g\left(M^{(2)}\right)-g(F)
$$

\subsection{Weak reduction of Heegaard splittings}

Let $M$ be a compact, orientable 3-manifold and $C_{1} \cup_{\Sigma} C_{2}$ a Heegaard splitting of $M$. Let $\Delta=\Delta_{1} \cup \Delta_{2}$ be a weakly reducing collection of disks for $\Sigma$, i.e., $\Delta_{i}(i=1,2)$ is a union of mutually disjoint, non-empty meridian disks for $C_{i}$ such that $\Delta_{1} \cap \Delta_{2}=\emptyset$. Then, $\Sigma(\Delta)$ denotes the surface obtained from $\Sigma$ by compressing along $\Delta$, i.e., $\Sigma(\Delta)=\left(\Sigma \backslash\left(N\left(\Delta_{1}, C_{1}\right) \cup N\left(\Delta_{2}, C_{2}\right)\right)\right) \cup$ $\operatorname{Fr}_{C_{1}} N\left(\Delta_{1}, C_{1}\right) \cup \operatorname{Fr}_{C_{2}} N\left(\Delta_{2}, C_{2}\right)$. Let $\widehat{\Sigma}(\Delta)$ be the surface obtained from $\Sigma(\Delta)$ by removing all the components that are contained in $C_{1}$ or $C_{2}$. We call $\widehat{\Sigma}(\Delta)$ the surface obtained from $\Sigma$ by weakly reducing along $\Delta$ (or the surface obtained from $\Sigma$ by a weak reduction along $\Delta$ ). The next lemma is well known (for a proof, see, for example, [8, Lemma 4.1]).

Lemma 2.10. If there is a 2-sphere component of $\widehat{\Sigma}(\Delta)$, then $C_{1} \cup_{\Sigma} C_{2}$ is reducible. 
Suppose that no component of $\widehat{\Sigma}(\Delta)$ is a 2-sphere. (By Lemma 2.10, we see that when $C_{1} \cup_{\Sigma} C_{2}$ is irreducible, this is always satisfied.) The following argument can be found in [1] (see also [8, Section 4]).

Let $M_{1}, \ldots, M_{n}$ be the closures of the components of $M \backslash \widehat{\Sigma}(\Delta)$, and let $M_{i, j}=M_{i} \cap C_{j},(i=1, \ldots, n, j=1,2)$. Then, we can show (for a proof, see, for example, [8, Lemma 4.3]):

Proposition 2.11. For each $j$, one of the following holds:

(1) $M_{j, 2} \cap \Sigma \subset \operatorname{int}\left(M_{j, 1} \cap \Sigma\right)$ and $M_{j, 1}$ is connected,

(2) $M_{j, 1} \cap \Sigma \subset \operatorname{int}\left(M_{j, 2} \cap \Sigma\right)$ and $M_{j, 2}$ is connected.

Suppose that $M_{j}$ satisfies the conclusion (1) ((2) resp.) of Proposition 2.11. Note that $M_{j, 1}\left(M_{j, 2}\right.$ resp.) is a compression body ((2) of Remarks 2.2). Let $C_{j, 1}=\operatorname{cl}\left(M_{j, 1} \backslash N\left(\partial_{+} M_{j, 1}, M_{j, 1}\right)\right)\left(C_{j, 2}=\operatorname{cl}\left(M_{j, 2} \backslash N\left(\partial_{+}\right.\right.\right.$ $\left.\left.M_{j, 2}, M_{j, 2}\right)\right)$ resp. $)$ and $C_{j, 2}=N\left(\partial_{+} M_{j, 1}, M_{j, 1}\right) \cup M_{j, 2}\left(C_{j, 1}=N\left(\partial_{+} M_{j, 2}\right.\right.$, $\left.M_{j, 2}\right) \cup M_{j, 1}$ resp. $)$. Note that $C_{j, 1}\left(C_{j, 2}\right.$ resp. $)$ is a compression body ((2) of Remarks 2.2) and that $C_{j, 2}\left(C_{j, 1}\right.$ resp.) is also a compression body by the definition of compression body. By using this, it is easy to see:

Proposition 2.12. Each $C_{i, j}$ is a compression body such that $C_{j, 1} \cup C_{j, 2}$ gives a Heegaard splitting of $M_{j}(j=1, \ldots, n)$ and that

$$
\left(C_{1,1} \cup C_{1,2}\right) \cup \cdots \cup\left(C_{n, 1} \cup C_{n, 2}\right)
$$

gives an untelescoping of $C_{1} \cup C_{2}$.

\subsection{Untelescoping and weak reduction}

Let $C_{1} \cup_{\Sigma} C_{2}$ be a Heegaard splitting of $M$ and $\left(C_{1}^{(1)} \cup_{\Sigma^{(1)}} C_{2}^{(1)}\right) \cup \cdots \cup$ $\left(C_{1}^{(n)} \cup_{\Sigma^{(n)}} C_{2}^{(n)}\right)$ an untelescoping of $C_{1} \cup_{\Sigma} C_{2}$. Let $R^{(i)}=C_{1}^{(i)} \cup C_{2}^{(i)}$.

Proposition 2.13. Suppose that there exists an $R^{(k)}$ such that a component of $\operatorname{Fr}_{M}\left(R^{(k)}\right)$, say $F$, is separating in $M$. Then, there exists a weak reducing collection of disks for $\Sigma$, say $\Delta=\Delta_{1} \cup \Delta_{2}$, so that $\widehat{\Sigma}(\Delta)$ is isotopic to $F$.

Proof. By exchanging superscripts if necessary, we may assume that $F$ separates $M$ into $R^{(1)} \cup \cdots \cup R^{(k)}$, say $M^{(1)}$, and $R^{(k+1)} \cup \cdots \cup R^{(n)}$, say $M^{(2)}$. Let $\mathcal{C}_{1}^{(1)} \cup \mathcal{C}_{2}^{(1)}\left(\mathcal{C}_{1}^{(2)} \cup \mathcal{C}_{2}^{(2)}\right.$ resp. $)$ be a Heegaard splitting of $M^{(1)}$ 
$\left(M^{(2)}\right.$ resp. $) \quad$ obtained from $\left(C_{1}^{(1)} \cup C_{2}^{(1)}\right), \ldots,\left(C_{1}^{(k)} \cup C_{2}^{(k)}\right)\left(\left(C_{1}^{(k+1)} \cup\right.\right.$ $\left.C_{2}^{(k+1)}\right), \ldots,\left(C_{1}^{(n)} \cup C_{2}^{(n)}\right)$ resp.) by a sequence of amalgamations. Then, let

$$
\begin{aligned}
& \left(\partial_{-} C_{1}^{(1)} \times[0,1]\right) \cup \mathcal{H}_{0}^{(1)} \cup \mathcal{H}_{1}^{(1)} \cup \mathcal{H}_{2}^{(1)} \cup \mathcal{H}_{3}^{(1)}, \\
& \left(\partial_{-} C_{1}^{(2)} \times[0,1]\right) \cup \mathcal{H}_{0}^{(2)} \cup \mathcal{H}_{1}^{(2)} \cup \mathcal{H}_{2}^{(2)} \cup \mathcal{H}_{3}^{(2)}
\end{aligned}
$$

be handle decompositions of $M^{(1)}$ and $M^{(2)}$, respectively, which can be used to amalgamate $\mathcal{C}_{1}^{(1)} \cup \mathcal{C}_{2}^{(1)}$ and $\mathcal{C}_{1}^{(2)} \cup \mathcal{C}_{2}^{(2)}$, i.e., $\mathcal{H}_{1}^{(2)} \cap\left(\mathcal{H}_{1}^{(1)} \cup \mathcal{H}_{2}^{(1)}\right)=\emptyset$ and $\mathcal{H}_{2}^{(2)} \cap \mathcal{H}_{2}^{(1)}=\emptyset$. Hence, the handle decomposition

$$
\begin{aligned}
& \left(\partial_{-} C_{1}^{(1)}\right) \cup\left(\left(\partial_{-} C_{1}^{(2)} \backslash F\right) \times[0,1]\right) \cup\left(\mathcal{H}_{0}^{(1)} \cup \mathcal{H}_{0}^{(2)}\right) \cup\left(\mathcal{H}_{1}^{(1)} \cup \mathcal{H}_{1}^{(2)}\right) \\
& \cup\left(\mathcal{H}_{2}^{(1)} \cup \mathcal{H}_{2}^{(2)}\right) \cup\left(\mathcal{H}_{3}^{(1)} \cup \mathcal{H}_{3}^{(2)}\right)
\end{aligned}
$$

corresponds to $C_{1} \cup C_{2}$. Then take a weak reducing collection $\Delta=\Delta_{1} \cup$ $\Delta_{2}$ of $C_{1} \cup C_{2}$ such that $\Delta_{1}$ corresponds to the co-cores of $\mathcal{H}_{1}^{(2)}$ and $\Delta_{2}$ corresponds to the cores of $\mathcal{H}_{2}^{(1)}$. It is easy to see that the weak reduction of $\Sigma$ along $\Delta$ yields a surface isotopic to $F$, thus proving Proposition 2.13.

\subsection{Minimal genus Heegaard splittings of (disk with $n$-holes) $\times S^{1}$}

Let $D(n)$ be the 3 -manifold (disk with $n$ holes) $\times S^{1}$ with $n \geq 2$. Let $T_{0}$, $T_{1}, \ldots, T_{n}$ be the boundary components of $D(n)$. For each pair of integers $p, q(\geq 0)$ with $p+q=n+1$ denote by $g_{p, q}$ the Heegaard genus of $D(n)$ partitioning the boundary into $p$ and $q$ components, that is,

$$
g_{p, q}=g\left(D(n) ; \bigcup_{i=0}^{p-1} T_{i}, \bigcup_{i=p}^{n} T_{i}\right) .
$$

The next proposition is required in Section 6 and is easily deduced from [26].

Proposition 2.14. $g_{0, n+1}=g_{n+1,0}=n+1$, otherwise $g_{p, q}=n$.

Moreover, by [26], we see that minimal genus Heegaard splittings are given as follows: let $Q$ be a disk with $n$ holes in $D(n)$, which is a cross-section of (disk with $n$ holes) $\times S^{1}$. Let $\alpha_{i}$ and $\beta_{i}$ be arcs properly embedded in $Q$ as in figure 1. Let $\partial_{0}, \partial_{1}, \ldots, \partial_{n}$ be boundary components of $Q$ such that $T_{i}=\partial_{i} \times S^{1}$. 


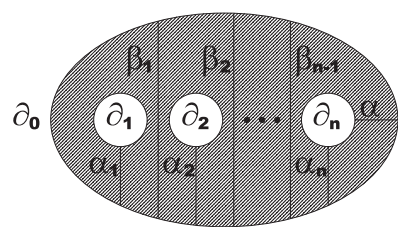

Figure 1: $D(n) \times S^{1}$.

Suppose that $\{p, q\}=\{0, n+1\}$. Let $C_{1}=N\left(\partial D(n) \cup\left(\cup_{i=1}^{n} \alpha_{i}\right)\right)$, and let $C_{2}=\operatorname{cl}\left(D(n) \backslash C_{1}\right)$. Then, $C_{1} \cup C_{2}$ gives a minimal genus Heegaard splitting of $(D(n) ; \partial D(n), \emptyset)$.

Suppose that $\{p, q\} \neq\{0, n+1\}$. Let $C_{1}=N\left(\left(\cup_{i=0}^{p-1} T_{i}\right) \cup\left(\cup_{i=1}^{p-1} \alpha_{i}\right) \cup\right.$ $\left.\left(\cup_{i=p}^{n-1} \beta_{i}\right)\right)$ and $C_{2}=\operatorname{cl}\left(D(n) \backslash C_{1}\right)$. Then, $C_{1} \cup C_{2}$ gives a minimal genus Heegaard splitting of $\left(D(n) ; \cup_{i=0}^{p-1} T_{i}, \cup_{i=p}^{n} T_{i}\right)$.

\section{Haken annuli}

In this section, we define Haken annuli and prove the basic facts about their behavior under amalgamation.

Definition 3.1. Let $C_{1} \cup_{\Sigma} C_{2}$ be a Heegaard splitting of $M$. An essential annulus $A \subset M$ is called a Haken annulus for $C_{1} \cup_{\Sigma} C_{2}$ if $A \cap \Sigma$ consists of a single simple closed curve that is essential in $A$.

\section{Remarks 3.2.}

(1) Note the similarity of Haken annulus to the Haken sphere [3] (see also [6, Chapter 5]) and the Haken disk [2].

(2) Let $D(n)$ be as in the end of Section 2. Observe that if $\{p, q\} \neq$ $\{0, n+1\}$, then there is a minimal genus Heegaard surface of $(D(n)$; $\left.\cup_{i=0}^{p-1} T_{i}, \cup_{i=p}^{n} T_{i}\right)$ with a Haken annulus of the form $\alpha \times S^{1}$, where $\alpha$ is an essential arc as in figure 1.

Given $C_{1} \cup_{\Sigma} C_{2}$, we described in Section 2 how to obtain a (not necessarily unique) handle decomposition for $M$ of the form

$$
M=\left(\partial_{-} C_{1} \times[0,1]\right) \cup \mathcal{H}^{0} \cup \mathcal{H}^{1} \cup \mathcal{H}^{2} \cup \mathcal{H}^{3},
$$

where $\mathcal{H}^{i}$ are handles of index $i$. 
We say that an annulus $A$ properly embedded in $M$ is vertical with respect to $C_{1} \cup_{\Sigma} C_{2}$ if $A \cap\left(\cup_{i=1}^{3} \mathcal{H}^{i}\right)=\emptyset$ (hence $\left.A \subset \partial_{-} C \times[0,1]\right), A$ is vertical in $\partial_{-} C \times[0,1]$ and $A \cap\left(\partial_{-} C_{1} \times\{0\}\right)$ is an essential simple closed curve in $\partial_{-} C_{1} \times\{0\}$.

From the definition of Haken annulus and Heegaard splitting induced by handle decomposition above, it is easy to see that:

Lemma 3.3. If $A$ is vertical with respect to $C_{1} \cup_{\Sigma} C_{2}$, then $A$ is a Haken annulus for the Heegaard splitting $C_{1} \cup_{\Sigma} C_{2}$.

Conversely, we have the following:

Lemma 3.4. Suppose that there exists a Haken annulus $A$ for $C_{1} \cup_{\Sigma} C_{2}$. Then, there exists a handle decomposition (with no 0- or 3-handles):

$$
M=\left(\partial_{-} C_{1} \times[0,1]\right) \cup \mathcal{H}^{1} \cup \mathcal{H}^{2}
$$

induced by $C_{1} \cup_{\Sigma} C_{2}$ such that $A \cap\left(\mathcal{H}^{1} \cup \mathcal{H}^{2}\right)=\emptyset$.

Proof. Since $C_{1}$ is a compression body, there exists a disjoint collection of meridian disks for $C_{1}$ (say $\mathcal{D}_{1}$ ) such that $C_{1}$ cut open along $\mathcal{D}_{1}$ is $\partial_{-} C_{1} \times$ $[0,1]$, where each component of $\mathcal{D}_{1}$ can be regarded as the co-core of a 1 handle for $C_{1}$. (Since $\partial_{-} C_{1} \neq \emptyset$, we may assume $C_{1}$ has no 0 -handles.)

Note that $A \cap C_{1}$ is a vertical annulus in $C_{1}$. Then by applying a standard innermost loop, outermost arc arguments to $\mathcal{D}_{1}$ and $A \cap C_{1}$, we can replace the collection $\mathcal{D}_{1}$ (without renaming it) so that $\left(A \cap C_{1}\right) \cap \mathcal{D}_{1}=\emptyset$. By using the same argument, we see that there exists a set of compressing disks for $C_{2}$ (say $\left.\mathcal{D}_{2}\right)$ such that $\mathcal{D}_{2} \cap\left(A \cap C_{2}\right)=\emptyset$ and $\mathcal{D}_{2}$ cuts $C_{2}$ into $\partial_{-} C_{2} \times[0,1]$ (since there exists a Haken annulus, $\partial_{-} C_{2} \neq \emptyset$, hence we may assume no component of $C_{2}$ cut along $\mathcal{D}_{2}$ is a 3-ball). Each component of $\mathcal{D}_{2}$ can be regarded as a core of a 2-handle for $C_{2}$. Then by taking the handle decomposition for $M$ obtained by $\mathcal{D}_{1}$ and $\mathcal{D}_{2}$, we obtain the conclusion of Lemma 3.4.

Suppose that $M$ is the union of two compact orientable manifolds (say $\left.M=M^{(1)} \cup M^{(2)}\right)$ such that $M^{(1)} \cap M^{(2)}=\partial M^{(1)} \cap \partial M^{(2)}=F$, a collection of components of $\partial M^{(i)}$. Let $C_{1}^{(1)} \cup_{\Sigma^{(1)}} C_{2}^{(1)}$ and $C_{1}^{(2)} \cup_{\Sigma^{(2)}} C_{2}^{(2)}$ be Heegaard splittings of $M^{(1)}$ and $M^{(2)}$, respectively. Let $F^{(1)} \cup F_{1}^{(1)} \cup F_{2}^{(1)}$ and $F^{(2)} \cup F_{1}^{(2)} \cup F_{2}^{(2)}$ be partitions of the components of $\partial M^{(1)}, \partial M^{(2)}$, 
respectively, such that the surfaces $F^{(1)}$ and $F^{(2)}$ are identified in $M$ as $F$, and

$$
\begin{aligned}
& \partial_{-} C_{1}^{(1)}=F_{1}^{(1)}, \quad \partial_{-} C_{2}^{(1)}=F^{(1)} \cup F_{2}^{(1)}, \\
& \partial_{-} C_{1}^{(2)}=F^{(2)} \cup F_{1}^{(2)}, \quad \partial_{-} C_{2}^{(2)}=F_{2}^{(2)} .
\end{aligned}
$$

Let $C_{1} \cup_{\Sigma} C_{2}$ be an amalgamation of $C_{1}^{(1)} \cup_{\Sigma^{(1)}} C_{2}^{(1)}$ and $C_{1}^{(2)} \cup_{\Sigma^{(2)}} C_{2}^{(2)}$.

Proposition 3.5. Let $M, M^{(1)}, M^{(2)}, C_{1} \cup_{\Sigma} C_{2}, C_{1}^{(1)} \cup_{\Sigma^{(1)}} C_{2}^{(1)}$ and $C_{1}^{(2)}$ $\cup_{\Sigma^{(2)}} C_{2}^{(2)}$ be as above. Suppose that there exists a Haken annulus $A^{(1)}$ for $C_{1}^{(1)} \cup_{\Sigma^{(1)}} C_{2}^{(1)}$ such that $A^{(1)} \cap F^{(1)}=\emptyset$. Then, the image of $A^{(1)}$ is a Haken annulus for $C_{1} \cup_{\Sigma} C_{2}$.

Proof. By Lemma 3.4, there exists a handle decomposition of $M^{(1)}$ of the form

$$
M^{(1)}=\left(\partial_{-} C_{1}^{(1)} \times[0,1]\right) \cup \mathcal{H}_{1}^{(1)} \cup \mathcal{H}_{2}^{(1)}
$$

induced by $C_{1}^{(1)} \cup_{\Sigma^{(1)}} C_{2}^{(1)}$ such that $A^{(1)} \cap\left(\mathcal{H}_{1}^{(1)} \cup \mathcal{H}_{2}^{(1)}\right)=\emptyset$. By the definition of amalgamation, we can take a handle decomposition

$$
M^{(2)}=\left(F^{(2)} \times[0,1]\right) \cup\left(F_{1}^{(2)} \times[0,1]\right) \cup \mathcal{H}_{0}^{(2)} \cup \mathcal{H}_{1}^{(2)} \cup \mathcal{H}_{2}^{(2)} \cup \mathcal{H}_{3}^{(2)}
$$

induced by $C_{1}^{(2)} \cup_{\Sigma^{(2)}} C_{2}^{(2)}$ such that these handles give the handle decomposition

$$
\begin{aligned}
M= & \left(F_{1}^{(1)} \times[0,1]\right) \cup\left(F_{1}^{(2)} \times[0,1]\right) \cup \mathcal{H}_{0}^{(2)} \cup\left(\mathcal{H}_{1}^{(1)} \cup \mathcal{H}_{1}^{(2)}\right) \\
& \cup\left(\mathcal{H}_{2}^{(1)} \cup \mathcal{H}_{2}^{(2)}\right) \cup \mathcal{H}_{3}^{(2)},
\end{aligned}
$$

which gives the Heegaard splitting $C_{1} \cup_{\Sigma} C_{2}$. Here we note that adding $F_{1}^{(2)} \times[0,1]$ and $\mathcal{H}_{i}^{(2)}$ does not affect $A^{(1)}$, since $A^{(1)} \cap F=\emptyset$. Hence we see that $A^{(1)} \cap\left(\cup_{i, j} \mathcal{H}_{i}^{(j)}\right)=\emptyset$, and this together with Lemma 3.3 shows that the image of $A^{(1)}$ is a Haken annulus for $C_{1} \cup_{\Sigma} C_{2}$.

Proposition 3.6. Let $M, M^{(1)}, M^{(2)}, C_{1} \cup_{\Sigma} C_{2}, C_{1}^{(1)} \cup_{\Sigma^{(1)}} C_{2}^{(1)}$ and $C_{1}^{(2)}$ $\cup_{\Sigma^{(2)}} C_{2}^{(2)}$ be as above. Suppose that there exists a Haken annulus $A^{(j)}$ for $C_{1}^{(j)} \cup_{\Sigma^{(j)}} C_{2}^{(j)}$ for each $j=1,2$ such that $A^{(1)} \cap F=A^{(2)} \cap F$ (hence the image of $A^{(1)} \cup A^{(2)}$ is an annulus properly embedded in $M$ ). Then, the image of $A^{(1)} \cup A^{(2)}$ is a Haken annulus for $C_{1} \cup_{\Sigma} C_{2}$. 
Proof. By Lemma 3.4, there exists a handle decomposition $\left(F_{1}^{(1)} \times[0,1]\right) \cup$ $\mathcal{H}_{1}^{(1)} \cup \mathcal{H}_{2}^{(1)}\left(\left(F_{1}^{(2)} \times[0,1]\right) \cup\left(F^{(2)} \times[0,1]\right) \cup \mathcal{H}_{1}^{(2)} \cup \mathcal{H}_{2}^{(2)}\right.$ resp. $)$ induced by $C_{1}^{(1)} \cup_{\Sigma^{(1)}} C_{2}^{(1)}\left(C_{1}^{(2)} \cup_{\Sigma^{(2)}} C_{2}^{(2)}\right.$ resp. $) \quad$ such that $A^{(1)} \cap\left(\mathcal{H}_{1}^{(1)} \cup \mathcal{H}_{2}^{(1)}\right)=\emptyset$ $\left(A^{(2)} \cap\left(\mathcal{H}_{1}^{(2)} \cup \mathcal{H}_{2}^{(2)}\right)=\emptyset\right.$ resp.). By pushing $F^{(2)} \times[0,1]$ into $M^{(1)}$, we obtain a handle decomposition $M=\left(F_{1}^{(1)} \times[0,1]\right) \cup\left(\mathcal{H}_{1}^{(1)} \cup \mathcal{H}_{2}^{(1)}\right) \cup\left(F_{1}^{(2)} \times\right.$ $[0,1]) \cup\left(\mathcal{H}_{1}^{(2)} \cup \mathcal{H}_{2}^{(2)}\right)$ (Section 2). Here, we may suppose that the image of $A^{(1)} \cup A^{(2)}$ is $A^{(1)}$. It is easy to see that by isotopy we may suppose, in addition to the above, that $\mathcal{H}_{1}^{(2)} \cap\left(\mathcal{H}_{1}^{(1)} \cup \mathcal{H}_{2}^{(1)}\right)=\emptyset$ and $\mathcal{H}_{2}^{(2)} \cap \mathcal{H}_{2}^{(1)}=\emptyset$. Hence, these handles give the Heegaard splitting $C_{1} \cup_{\Sigma} C_{2}$. For these handles, we have

$$
A^{(1)} \cap\left(\mathcal{H}_{1}^{(1)} \cup \mathcal{H}_{1}^{(2)} \cup \mathcal{H}_{2}^{(1)} \cup \mathcal{H}_{2}^{(2)}\right)=\emptyset
$$

Then, Lemma 3.3 establishes the conclusion of Proposition 3.6.

\section{The Swallow Follow Torus Theorem}

Let $X$ be the exterior of a knot $K$ in a closed orientable 3 -manifold. Let $\gamma_{1}, \ldots, \gamma_{p}$ be mutually disjoint simple closed curves that are obtained by pushing mutually disjoint meridional curves in $\partial X$ into int $(X)$. Then, let $X^{(p)}=\operatorname{cl}\left(X \backslash \cup_{i=1}^{p} N\left(\gamma_{i}\right)\right)$. Set $X^{(0)}=X$. A torus $T$ in $X^{(p)}$ is called a swallow follow torus if it is separating and essential in $X^{(p)}$, and there exists an annulus $A$ in $X^{(p)}$ so that $A \cap \partial X^{(p)}=\partial A \cap \partial X^{(p)}$ is a meridian curve in $\partial X$ and $A \cap T=\partial A \cap T$ is an essential simple closed curve in $T$. We call this annulus a meridionally compressing annulus for $T$. Let $A^{\prime}$ be the annulus properly embedded in $X^{(p)}$ obtained from $T$ by removing $N(A) \cap T$ and then adding two parallel copies of $A$. (We say that $A^{\prime}$ is obtained from $T$ by performing surgery along $A$.) Since $T$ is essential, we see that $A^{\prime}$ is also essential. The annulus $A^{\prime}$ gives a decomposition

$$
X_{1}^{\left(p_{1}\right)} \cup_{A^{\prime}} X_{2}^{\left(p_{2}\right)}, \quad p=p_{1}+p_{2},
$$

where each $X_{i}$ is the exterior of a knot $K_{i}$ (possibly the trivial knot in $S^{3}$, in which case $X_{i}^{\left(p_{i}\right)}$ is homeomorphic to (disk with $p_{i}$ holes) $\times S^{1}$; recall that this was denoted $D\left(p_{i}\right)$ at the end of Section 2). We call this decomposition the decomposition induced by $T$. Note that $T$ can be retrieved from $A^{\prime}$ by adding a component of $\partial X \backslash \partial A^{\prime}$ and applying an isotopy pushing it into int $X^{(p)}$. The component of $\partial X \backslash \partial A^{\prime}$ follows $K_{1}$ or $K_{2}$, say $K_{2}$. This yields 
the following decomposition:

$$
X^{(p)}=X_{1}^{\left(p_{1}+1\right)} \cup_{T} X_{2}^{\left(p_{2}\right)}
$$

The purpose of this section is to prove the following:

Theorem 4.1 (the Swallow Follow Torus Theorem). Let $K_{1}, \ldots, K_{n}$ $(n \geq 2)$ be knots in closed orientable 3-manifolds. Let $X_{1}, \ldots, X_{n}$ and $X$ be the exteriors of $K_{1}, \ldots, K_{n}$ and $\#_{i=1}^{n} K_{i}$, respectively. Suppose that no $X_{i}$ is a solid torus and $X$ is irreducible. Then,

(1) For any $p \geq 0$, every Heegaard surface for $X^{(p)}$ weakly reduces to exactly one surface which is a swallow follow torus, or

(2) For some $i, X_{i}$ contains an essential meridional surface; more precisely, if a genus $g$ Heegaard surface for $X^{(p)}$ (for some $p$ ) does not weakly reduce to a swallow follow torus, then there exists a meridional essential surface $S \subset X_{i}$ so that $\chi(S) \geq 4-2 g\left(X^{(p)}\right)$.

Remark 4.2. One might hope that Theorem 4.1 holds for $n=1$. However, this is not the case. Let $K$ be a non-trivial 2-bridge knot in $S^{3}$. Then, $K$ is m-small [4] and $g(X)=g\left(X^{(1)}\right)=2$ (see, for example, [14]). If a genus 2 Heegaard surface weakly reduces, then the obtained essential surface is a 2-sphere. Applying this to $X^{(1)}$, we see that (since $X^{(1)}$ is irreducible) every minimal genus Heegaard splitting of $X^{(1)}$ is strongly irreducible; hence the conclusion of Theorem 4.1 does not hold for $X^{(1)}$.

As an immediate consequence of Theorem 4.1, we have:

Corollary 4.3. Let $K_{i}, K, X_{i}$ and $X$ be as in the statement of Theorem 4.1, and suppose that each $K_{i}$ is $m$-small. Then, for any $p \geq 0$, every Heegaard splitting of $X^{(p)}$ weakly reduces to a swallow follow torus.

Proof of Theorem 4.1. Note that if some $K_{i}$ is a composite knot then $X_{i}$ contains an essential meridional annulus and conclusion (2) of Theorem 6.3 holds. We assume from now on that for each $i, K_{i}$ is prime.

Claim 4.4. Either existence and uniqueness of prime factorization holds for $K$ or conclusion (2) holds.

We note that prime factorization of knots in 3-manifolds is studied by Miyazaki in [11] and it is shown that neither existence nor uniqueness holds in general. 
Proof of Claim 4.4. It is shown in the existence theorem of [11] that if the meridian of a knot $K$ is non-null homotopic, then the number of prime factors of $K$ is unique. If the meridian of $K$ is null homotopic, then by the loop theorem (e.g., [5]), $\partial X$ is compressible, and either $X$ is a solid torus or it is reducible, both contrary to our assumptions. The uniqueness theorem of [11] implies that if the uniqueness of prime decomposition of $K$ fails, then the exterior of some factor of $K$ contains a non-separating meridional annulus; this gives conclusion (2).

By Claim 4.4, for the remainder of the proof, we may suppose that $K$ has a unique prime factorization.

Note that if a given Heegaard splitting weakly reduces to a swallow follow torus, then every stabilization of that Heegaard splitting can be weakly reduced to the same swallow follow torus; it therefore suffices to prove the theorem for non-stabilized Heegaard splittings. Fix $p$ and let $C_{1} \cup_{\Sigma} C_{2}$ be a non-stabilized Heegaard splitting of $X^{(p)}$.

Let $\mathcal{A}=A_{1} \cup \cdots \cup A_{n}$ be a union of mutually disjoint annuli properly embedded in $X^{(p)}$ with the following properties:

(1) For each $i, \partial A_{i} \subset \partial X$,

(2) $X^{(p)}$ cut open along $\mathcal{A}$ consists of $n+1$ components, $n$ homeomorphic to $X_{1}, \ldots, X_{n}$ (by slightly abusing notation $X_{i}$ will refer to the component homeomorphic to $X_{i}$ ) and one component homeomorphic to $D(p)$ (as defined in the end of Section 2), denoted $X_{0}$, and

(3) For each $i, X_{i} \cap \mathcal{A}=A_{i}$.

We say that the annuli of $\mathcal{A}$ are not nested. Let $T_{j}(j=1, \ldots, p)$ denote the component of $\partial X^{(p)}$ corresponding to $\partial N\left(\gamma_{j}\right)$. (Hence $\partial X^{(p)}=\partial X \cup$ $\left(\cup_{j=1}^{p} T_{j}\right)$.)

We divide the proof into the following cases.

Case 1. $\Sigma$ is strongly irreducible. Since $\Sigma$ is strongly irreducible, we can isotope it to intersect $\mathcal{A}$ in simple closed curves that are essential in both $\mathcal{A}$ and $\Sigma$. Minimize $|\mathcal{A} \cap \Sigma|$ subject to that constraint. Let $\Sigma_{i}=\Sigma \cap X_{i}$ $(i=0,1, \ldots, n)$. Suppose that $\Sigma_{i}=\emptyset$ for some $i(1 \leq i \leq n)$. Then, $A_{i}$ is contained in a compression body $C_{j}(j=1$, or 2$)$. By (6) of Remarks 2.2, we see that $A_{i}$ is boundary parallel in $C_{j}$. This shows that $X_{i}$ is a solid torus, contradicting the assumption of Theorem 4.1. Hence, $\Sigma_{i} \neq \emptyset$ for each $i(i=1, \ldots, n)$. 
The proof of the following claim is similar to the proof of Claim 4 in page 247 of [9]; however, for the convenience of the reader, we include it here.

\section{Claim 4.5.}

(1) There exists $i$ (possibly $i=0$ ) such that $\Sigma_{i}$ is compressible in $X_{i}$, and there exist two compressing disks $D_{1}, D_{2} \subset X_{i}$ that compress $\Sigma_{i}$ into the $C_{1}$ side and $C_{2}$ side, respectively, and $D_{1} \cap D_{2} \neq \emptyset$.

(2) For each $k \neq i, \Sigma_{k}$ is incompressible in $X_{k}$.

Proof of Claim 4.5. Note that since $\Sigma \cap \mathcal{A}$ is essential in $\mathcal{A}$, any compressing disk for $\Sigma_{i}$ is also a compressing disk for $\Sigma$.

Subclaim. For each $i=1,2$, there is a meridional disk $D_{i}$ of $C_{i}$ so that $D_{i} \cap \mathcal{A}=\emptyset$.

Proof. Fix $i$ and let $D_{i} \subset C_{i}$ be a meridian disk that minimizes $\left|D_{i} \cap \mathcal{A}\right|$. If $D_{i} \cap \mathcal{A}=\emptyset$, we are done. Else, by minimality of $\left|D_{i} \cap \mathcal{A}\right|$ and a standard innermost disk argument, no component of $D_{i} \cap \mathcal{A}$ is a simple closed curve, and each outermost disk in $D_{i}$ gives a boundary compression of $\Sigma_{k}$ in $X_{k}$ (for some $k$ ). By Lemma 2.1, we see that either there is a compression of $\Sigma_{k}$ in $X_{k}$ which is contained in $C_{i}$ or a component of $\Sigma_{k}$ is a boundary parallel annulus in $X_{k}$; the former is the desired conclusion of the subclaim, and the latter contradicts minimality of $\left|D_{i} \cap \mathcal{A}\right|$, establishing the subclaim.

Let $D_{1}, D_{2}$ be as in Subclaim. If $D_{1} \cap D_{2}=\emptyset$, then $\left\{D_{1}, D_{2}\right\}$ gives a weak reduction of $\Sigma$, contrary to our assumption. Hence, $D_{1}$ and $D_{2}$ are contained in the same component ( say $X_{i}$ ). Finally note that if for $k \neq i$ $\Sigma_{k}$ compresses in $X_{k}$, then the compressing disk for $\Sigma_{k}$ and either $D_{1}$ or $D_{2}$ gives a weak reduction of $\Sigma$, a contradiction; hence, for each $k \neq i, \Sigma_{k}$ is incompressible.

Since $n \geq 2$, we may suppose that $\Sigma_{1}$ satisfies (2), i.e., $\Sigma_{1}$ is incompressible in $X_{1}$. Let $\Sigma^{\prime}$ be the surface obtained by compressing $\Sigma$ along $D_{1}$. Since $\mathcal{A}$ is incompressible and $\mathcal{A} \cap \Sigma$ is essential in $\mathcal{A}$, no component of $\Sigma^{\prime}$ cut open along $\mathcal{A}$ has positive Euler characteristic. Hence, $\chi\left(\Sigma_{1}\right)=$ $\chi\left(\Sigma^{\prime} \cap X_{1}\right) \geq 4-2 g$. Suppose that each component of $\Sigma_{1}$ is boundary parallel in $X_{1}$. Then the minimality of $|\mathcal{A} \cap \Sigma|$ implies that each component of $\Sigma_{1}$ is parallel in $X_{1}$ to the annulus $X_{1} \cap \partial X$. Let $X_{1}^{\prime}=\operatorname{cl}\left(X_{1} \backslash N\left(\partial X_{1}, X_{1}\right)\right)$. Then, we can push $\Sigma \cap X_{1}^{\prime}$ out of $X_{1}^{\prime}$; hence, we may assume $X_{1}^{\prime} \cap \Sigma=\emptyset$, and $X_{1}^{\prime}$ is contained in a compression body $C_{j}(j=1$, or 2$)$. Note that $\partial X_{1}^{\prime}$ is incompressible in $X$ and therefore in $C_{j}$. Hence $\partial X_{1}^{\prime}$ is parallel to $\partial_{-} C_{j}$ 
((6) of Remarks 2.2). This shows that $X^{(p)}$ is homeomorphic to $X_{1}^{\prime}$, but this contradicts the assumption that $n \geq 2$. Hence, some component of $\Sigma_{1}$ is not boundary parallel in $X_{1}$. This establishes conclusion (2) of Theorem 4.1.

Case 2. $\Sigma$ is weakly reducible. Take a S-T untelescoping (Section 2) of $C_{1} \cup_{\Sigma} C_{2}$ to obtain a (possibly disconnected) incompressible surface $S$ (corresponding to the union of $\partial R_{j}$ in Remark 2.5 minus $\left.\partial X^{(p)}\right)$ with $\chi(S) \geq$ $6-2 g$. Denote the connected components of $S$ by $S_{j}$. Since $C_{1} \cup_{\Sigma} C_{2}$ is non-stabilized and $X$ is irreducible, we see, by Proposition 2.13, Lemma 2.10 and Waldhausen's classification of Heegaard surfaces of $S^{3}$ [28], that no $S_{j}$ is a 2 -sphere. Hence, for each $j, \chi\left(S_{j}\right) \geq 6-2 g$. Since both $S$ and $\mathcal{A}$ are incompressible, we may suppose that each component of $S \cap \mathcal{A}$ is a simple closed curve which is essential in both $S$ and $\mathcal{A}$. Minimize $|S \cap \mathcal{A}|$ subject to this constraint.

We have the following subcases.

Subcase 2a. $S \cap \mathcal{A}=\emptyset$. Let $M$ be the component of $X^{(p)}$ cut open along $S$ containing $\partial X$. Note that in this case $\mathcal{A} \subset M$. Let $C_{M, 1} \cup_{\Sigma_{M}} C_{M, 2}$ be the Heegaard splitting of $M$ induced from $\Sigma$. Since $\mathcal{A} \subset M, M$ is not a product $\partial X \times[0,1]$. Since $C_{M, 1} \cup_{\Sigma_{M}} C_{M, 2}$ is strongly irreducible, we may suppose each component of $\Sigma_{M} \cap \mathcal{A}$ is essential in both $\Sigma_{M}$ and $\mathcal{A}$; minimize $\left|\Sigma_{M} \cap \mathcal{A}\right|$ subject to this constraint.

Then we claim that $\Sigma_{M} \cap \mathcal{A} \neq \emptyset$. Suppose, for a contradiction, that $\Sigma_{M} \cap \mathcal{A}=\emptyset$. Then by (6) of Remarks 2.2, we have that each $A_{i}$ is boundary parallel in $C_{M, 1}$. Hence, each $A_{i}$ is boundary parallel in $X^{(p)}$. This shows that each $X_{i}$ is a solid torus, contradicting the assumption of Theorem 4.1.

By Claim 4.5, we may assume that each component of $\Sigma_{M} \cap\left(X_{1} \cap M\right)$ $\left(=\Sigma_{M} \cap X_{1}\right)$ is incompressible in $X_{1} \cap M$. Note that each component of $\operatorname{Fr}_{X^{(p)}}\left(X_{1} \cap M\right)$ is either $A_{1}$ or a component of $S$ and therefore is incompressible in $X_{1}$. This shows that each component of $\Sigma_{M} \cap X_{1}$ is incompressible in $X_{1}$.

If some component of $\Sigma_{M} \cap X_{1}$ is not boundary parallel in $X_{1}$, then we have conclusion (2) of Theorem 4.1. Suppose that each component of $\Sigma_{M} \cap X_{1}$ is a boundary parallel annulus in $X_{1}$. Pick a component of $\Sigma_{M} \cap X_{1}$ and let $V$ be the product region that it separates from $X_{1}$; note that $V$ is a solid torus. Since each component of $S$ is incompressible in $X$, we see that no component of $S$ is contained in $V$. Let $X_{1}^{\prime}=\operatorname{cl}\left(X_{1} \backslash N\left(\partial X_{1}, X_{1}\right)\right)$. Since $S \cap V=\emptyset$, we can push $\Sigma_{M} \cap X_{1}^{\prime}$ out of $X_{1}^{\prime}$. Hence we have $\partial X_{1}^{\prime} \subset$ $C_{M, 1}$ or $\partial X_{1}^{\prime} \subset C_{M, 2}$, say $C_{M, 1}$. Since $\partial X_{1}^{\prime}$ is incompressible in $X$, it is incompressible in $C_{M, 1}$. Hence, $\partial X_{1}^{\prime}$ is parallel to a component of $\partial_{-} C_{M, 1}$ 
((6) of Remarks 2.2). Note that each component of $\partial_{-} C_{M, 1}$ is either $\partial X$ or a component of $S$. Since $X \neq X_{1}$, we see that $\partial X_{1}^{\prime}$ is parallel to a component of $S$. By Proposition 2.13, there is a weak reduction of $C_{1} \cup_{\Sigma} C_{2}$ which gives exactly $\partial X_{1}^{\prime}$. This establishes conclusion (1) of Theorem 4.1.

Subcase 2b. $\quad S \cap \mathcal{A} \neq \emptyset$. Suppose that the conclusion (2) of Theorem 4.1 does not hold; hence, for each $i \neq 0$, each component of $S \cap X_{i}$ is closed or a boundary parallel annulus.

Let $X_{i}^{\prime}=\operatorname{cl}\left(X_{i} \backslash N\left(\partial X_{i}, X_{i}\right)\right)(i=1, \ldots, n)$ and $X_{0}^{\prime}=\operatorname{cl}\left(X \backslash \cup_{i=1}^{n} X_{i}^{\prime}\right)$. Note that $X_{0}^{\prime}$ is homeomorphic to a (disk with $n+p$ holes) $\times S^{1}$. Since each component of $S \cap X_{i}$ with non-empty boundary is a boundary parallel annulus, they can be pushed into $X_{0}^{\prime}$. Hence, we have $S \cap \partial X_{0}^{\prime}=\emptyset$, and $S \cap X_{0}^{\prime} \neq \emptyset$. Since $S$ is a closed incompressible surface in the Seifert fibered space $X_{0}^{\prime}$, it must be vertical by [6, Theorem VI.34]; that is to say, $S$ is isotopic to a surface of the form (simple closed curve $(\mathrm{s})) \times S^{1}$. Note that any essential simple closed curve on a disk with $n+p$ holes is separating. Hence, each component of $S \cap X_{0}^{\prime}$ is separating in $X_{0}^{\prime}$ and therefore also in $X^{(p)}$. Suppose that there is a component of $S \cap X_{0}^{\prime}$, say $S_{0}$, which is not boundary parallel in $X^{(p)}$. Then $S_{0}$ separates $X_{0}^{\prime}$ into two components, and let $X_{0}^{\prime \prime}$ denote the closure of the component of $X_{0} \backslash S_{0}$ such that $\partial X \subset X_{0}^{\prime \prime}$. Let $A$ be a vertical annulus properly embedded in $X_{0}^{\prime \prime}$ such that one component of $\partial A$ is contained in $\partial X$ and the other in $S_{0}$. Note that $A \cap \partial X$ is a meridian curve and $A \cap S_{0}$ is an essential simple closed curve in $S_{0}$. Hence, $S_{0}$ is a swallow follow torus in $X^{(p)}$. Finally, by Proposition 2.13, we see that a weak reduction of $C_{1} \cup_{P} C_{2}$ gives $S_{0}$. This establishes conclusion (1) of Theorem 4.1.

Finally suppose that each component of $S \cap X_{0}^{\prime}$ is boundary parallel in $X^{(p)}$. Let $\widetilde{P}$ be the union of the parallel regions bounded by the components of $S \cap X_{0}^{\prime}$. Then, let $\widetilde{X}^{(p)}=\operatorname{cl}\left(X^{(p)} \backslash \widetilde{P}\right)$ and $\widetilde{C}_{1} \cup_{\tilde{\Sigma}} \widetilde{C}_{2}$ be the Heegaard splitting of $\widetilde{X}^{(p)}$ obtained from the $\mathrm{S}-\mathrm{T}$ untelescoping of $C_{1} \cup_{\Sigma} C_{2}$ by amalgamating the pieces contained in $\widetilde{X}^{(p)}$. Then, we apply the above argument to $\widetilde{C}_{1} \cup_{\widetilde{\Sigma}} \widetilde{C}_{2}$, that is, we apply the argument of Case 1 if $\widetilde{\Sigma}$ is strongly irreducible or the argument of Case 2 is $\widetilde{\Sigma}$ is weakly reducible. Note that for $\widetilde{\Sigma}$, we do not have the case corresponding to Subcase $2 \mathrm{~b}$ with each component of $S \cap X_{0}^{\prime}$ being boundary parallel in $X^{(p)}$. Then, we have either conclusion (1) or conclusion (2) for $\widetilde{C}_{1} \cup_{\widetilde{\Sigma}} \widetilde{C}_{2}$. Conclusion (2) for $\widetilde{\Sigma}$ immediately establishes conclusion (2) for $C_{1} \cup_{\Sigma} C_{2}$. If we have conclusion (1) for $\widetilde{C}_{1} \cup_{\tilde{\Sigma}} \widetilde{C}_{2}$, then this together with Proposition 2.13 establishes conclusion (1) for $C_{1} \cup_{\Sigma} C_{2}$.

This completes the proof of Theorem 4.1. 


\section{Numerical bounds}

Theorem 5.1. Let $X$ be the exterior of a knot $K$ in a closed orientable 3-manifold. Suppose that there exists a minimal genus Heegaard surface for $X$ that weakly reduces to a swallow follow torus inducing the decomposition $K=K_{1} \# K_{2}$; let $X_{i}=E\left(K_{i}\right)$. Then, we have

$$
g\left(X_{1}\right)+g\left(X_{2}\right)-1 \leq g(X) \leq g\left(X_{1}\right)+g\left(X_{2}\right) .
$$

Remark 5.2. Recall from Section 1 that the right-hand side inequality always holds.

As a consequence of Theorem 5.1 and Corollary 4.3, we have:

Corollary 5.3. Let $K_{1}, \ldots, K_{n}$ be m-small knots in closed orientable 3manifolds, let $X_{i}=E\left(K_{i}\right)$, and $X=E\left(\#_{i=1}^{n} K_{i}\right)$. Suppose that no $X_{i}$ is a solid torus and $X$ is irreducible. Then, we have the following inequalities:

$$
\sum_{i=1}^{n} g\left(X_{i}\right)-(n-1) \leq g(X) \leq \sum_{i=1}^{n} g\left(X_{i}\right)
$$

Proof of Theorem 5.1. By Section 4, we have a decomposition: $X=X_{1}^{(1)} \cup$ $X_{2}$ or $X=X_{1} \cup X_{2}^{(1)}$. Since the argument is symmetric, we may suppose that $X=X_{1}^{(1)} \cup X_{2}$.

Note that $X_{1}$ is obtained from $X_{1}^{(1)}$ by Dehn filling and that the core of the attached solid torus is parallel to a meridian curve on $\partial X_{1}$. Since any Heegaard surface for $X_{1}$ can be regarded as the frontier of a compression body obtained from $N(\partial X, X)$ by attaching 1-handles, this implies that the core of the attached solid torus, say $l$, is isotopic into every Heegaard surface of $X_{1}$, that is to say, the filling is called good, as in [19, Definition 4.1] (see also $[20,21]$ ). Let $C_{1}^{\prime} \cup_{\Sigma^{\prime}} C_{2}^{\prime}$ be a minimal genus Heegaard splitting of $X_{1}$ such that $\partial X_{1} \subset C_{1}^{\prime}$, and $l \subset C_{2}^{\prime}$. Let $\widetilde{\Sigma}^{\prime}$ be a surface obtained from $\Sigma$ and $\partial N(l)$ as is shown in [19, Corollary 4.3]; note that $g\left(\widetilde{\Sigma}^{\prime}\right)=g\left(\Sigma^{\prime}\right)+1$ (in fact, $\widetilde{\Sigma}^{\prime}$ is a stabilization of $\left.\Sigma^{\prime}\right)$ and $\widetilde{\Sigma}^{\prime}$ is a Heegaard surface for $X_{1}^{(1)}$. Thus, we get the inequality $g\left(X_{1}^{(1)}\right) \leq g\left(X_{1}\right)+1$. Since $X_{1}$ is obtained from $X_{1}^{(1)}$ by Dehn filling, we immediately see that $g\left(X_{1}\right) \leq g\left(X_{1}^{(1)}\right)$. Combining the two, we have

$$
g\left(X_{1}\right) \leq g\left(X_{1}^{(1)}\right) \leq g\left(X_{1}\right)+1
$$


On the other hand, by Proposition 2.9, we have the following equality:

$$
g(X)=g\left(X_{1}^{(1)}\right)+g\left(X_{2}\right)-1 .
$$

Therefore, we have

$$
g\left(X_{1}\right)+g\left(X_{2}\right)-1 \leq g(X) \leq g\left(X_{1}\right)+g\left(X_{2}\right) .
$$

In Section 7, we will need the following information which follows from the proof of Theorem 5.1, and so we single it out here.

Corollary 5.4. If a minimal genus Heegaard splitting for $X$ weakly reduces to a swallow follow torus $T$ inducing $X=X_{1}^{(1)} \cup_{T} X_{2}$, then either $g\left(X_{1}\right)=$ $g\left(X_{1}^{(1)}\right)$ or $g\left(X_{1}\right)=g\left(X_{1}^{(1)}\right)-1$, and exactly one of the following holds:

(1) $g(X)=g\left(X_{1}\right)+g\left(X_{2}\right)$ and $g\left(X_{1}\right)=g\left(X_{1}^{(1)}\right)-1$.

(2) $g(X)=g\left(X_{1}\right)+g\left(X_{2}\right)-1$ and $g\left(X_{1}\right)=g\left(X_{1}^{(1)}\right)$.

\section{Hopf-Haken annulus theorem}

Let $K_{1}, \ldots, K_{n}(n \geq 1)$ be knots in closed orientable 3 -manifolds. Let $X_{1}, \ldots, X_{n}$, and $X$ be the exteriors of $K_{1}, \ldots, K_{n}$, and $K=\#_{i=1}^{n} K_{i}$, respectively.

Recall from Section 4 that for $p \geq 1, X^{(p)}=\operatorname{cl}\left(X \backslash \cup_{i=1}^{p} N\left(\gamma_{i}\right)\right)$, where $\gamma_{1}, \ldots, \gamma_{p}$ are simple closed curves obtained by pushing mutually disjoint meridian curves in $\partial X$ into int $X$ (with $\left.X^{(0)}=X\right)$. One can think of $X^{(p)}$ as the exterior of the link obtained from $K$ by taking connected sum with the Hopf link $n$ times. Note that the exterior of the Hopf link has an essential annulus connecting the distinct boundary components, where one boundary component of the annulus is a meridian curve, and the other is a longitude. This shows that $X^{(p)}$ has an essential annulus as well (provided $p \geq 1$ ) and motivates the following definition (cf. Definition 3.1):

Definition 6.1 (Hopf-Haken annulus). A Hopf spanning annulus in $X^{(p)}$ is a properly embedded essential annulus $A$ with one component of $\partial A$ a meridian curve of $\partial X$ and the other component a longitude of $\partial N\left(\gamma_{i}\right)$ for some $i$. If, in addition, there exists a Heegaard splitting $C_{1} \cup_{\Sigma} C_{2}$ of $X^{(p)}$ such that $A$ intersects $\Sigma$ in one simple closed curve that is essential in $A$, then $A$ is called a Hopf-Haken annulus for $C_{1} \cup_{\Sigma} C_{2}$. In that case, $\Sigma$ is said to admit a Hopf-Haken annulus. 


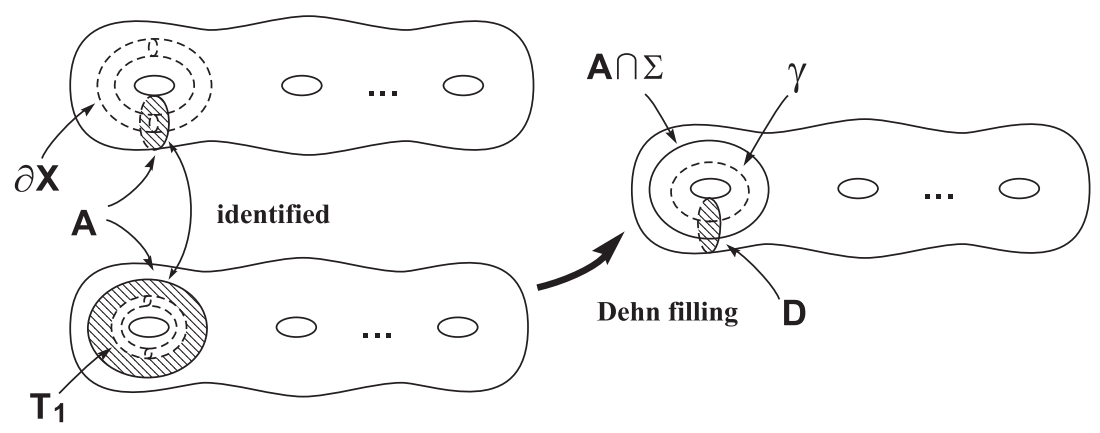

Figure 2: A primitive meridian.

By figure 2, we immediately have the following.

Proposition 6.2. Let $Y$ be the exterior of a knot $K$. Suppose that for some $p \geq 1$ there exists a Heegaard surface $\Sigma$ of $Y^{(p)}$ such that $g(\Sigma)=g(Y)$ and $\Sigma$ admits a Hopf-Haken annulus. Then $K$ admits a primitive meridian.

In this section, we prove the following theorem.

Theorem 6.3 (The Hopf-Haken annulus theorem). Let $K_{i}(i=1, \ldots$, $n, n \geq 1)$ be knots in closed orientable 3-manifolds. Let $X_{i}$ and $X$ be the exteriors of $K_{i}$, and $K=\#_{i=1}^{n} K_{i}$, respectively. Let $X^{(p)}$ be as above. Suppose that no $X_{i}$ is a solid torus and $X$ is irreducible. Then, one of the following holds.

(1) For each $p(\geq 1)$, there exists a minimal genus Heegaard surface of $X^{(p)}$ that admits a Hopf-Haken annulus.

(2) For some $i(1 \leq i \leq n), X_{i}$ contains a meridional essential surface $S$ such that $\chi(S) \geq 4-2 g$, where $g=g\left(X^{(p)}\right)$.

Remark 6.4. Note that in Theorem 6.3 we cannot expect every minimal genus Heegaard surface to satisfy conclusion (1), even when conclusion (2) is not satisfied. To see this, consider the following example: let $K_{m}$ be the knots constructed by Morimoto-Sakuma-Yokota in [18] and $X_{m}$ their exteriors. Then, $g\left(X_{m}\right)=2$ (by [18]) and $g\left(X_{m}^{(1)}\right)=3$ (by [10]). There is an essential torus $T \subset X_{m}$ giving the decomposition $X_{m}^{(1)}=D(2) \cup X_{m}^{\prime}$, where $X_{m}^{\prime}$ is homeomorphic to $X_{m}$ and $D(2)$ is (disk with two holes) $\times S^{1}$. Note that $\partial D(2)=\partial X_{m}^{(1)} \cup T$. By Proposition 2.14 and the comment after it, $g(D(2))=2$, and there exists a genus two Heegaard splitting of $D(2)$ 
(say $C_{1}^{\prime} \cup_{\Sigma^{\prime}} C_{2}^{\prime}$ ), with $\partial_{-} C_{1}^{\prime}=\partial X_{m}^{(1)}$ and $\partial_{-} C_{2}^{\prime}=T$. Let $C_{1} \cup_{\Sigma} C_{2}$ be the Heegaard splitting of $X_{m}^{(1)}$ obtained by amalgamating $C_{1}^{\prime} \cup_{\Sigma^{\prime}} C_{2}^{\prime}$ and a minimal genus Heegaard splitting of $X_{m}^{\prime}$. By Lemma 2.7, $C_{1} \cup_{\Sigma} C_{2}$ is a minimal genus Heegaard splitting of $X_{m}^{(1)}$. By the definition of amalgamation, we see that $\partial_{-} C_{1}=\partial X_{m}^{(1)}$. We conclude that $C_{1} \cup_{\Sigma} C_{2}$ does not admit a HopfHaken annulus, for if it did, then the components of $\partial X_{m}^{(1)}$ would be in distinct compression bodies.

Although we do not know if $K_{m}$ is m-small, this example seems to indicate that replacing "there exists" with "for every" in conclusion (1) of Theorem 6.3 is not likely to be possible. Note that the only property of $K_{m}$ used in the construction above is that $g\left(X^{(1)}\right)=g(X)+1$.

As consequences of Theorem 6.3, we obtain the following.

Corollary 6.5. Let $K_{i}, X_{i}, K, X$ and $X^{(p)}$ be as in the statement of Theorem 6.3. If $g\left(X^{(p)}\right)=g(X)$ for some $p \geq 1$, and no $X_{i}$ contains a meridional essential surface $S$ with $\chi(S) \geq 4-2 g(X)$, then $K$ admits a primitive meridian.

Corollary 6.6. Let $K_{i}, X_{i}, X$ and $X^{(p)}$ be as in the statement of Theorem 6.3. Suppose that no $X_{i}$ contains a meridional essential surface $S$ with $\chi(S) \geq 4-2 g\left(X^{(1)}\right)$. Then, $\left.g\left(X^{(1)}\right)=g\left(X^{(1)}, \partial X, \partial X^{(1)} \backslash \partial X\right)\right)$, i.e., there is a minimal genus Heegaard surface for $X^{(1)}$ separating the boundary components.

Proof of Corollary 6.5. By Theorem 6.3, there exists a minimal genus Heegaard splitting of $X^{(p)}$ (say $C_{1} \cup \Sigma_{\Sigma^{*}} C_{2}$ ) and a Hopf-Haken annulus $A^{*}$ for $\Sigma^{*}$. By assumption, $g\left(\Sigma^{*}\right)=g(X)$. Hence, by Proposition $6.2, K$ admits a primitive meridian.

Proof of Corollary 6.6. Our assumption implies that conclusion (2) of Theorem 6.3 cannot hold. With $p=1$, the minimal genus Heegaard surface for $X^{(1)}$ described in conclusion (1) of Theorem 6.3 separates the boundary components, thus proving the corollary.

Proof of Theorem 6.3. Recall from the proof of Theorem 4.1 that we may assume that each $K_{i}$ is a prime knot, and both existence and uniqueness of prime decomposition holds for $K$. We define the complexity of $X^{(p)}$ to be $(n, p)$ with the lexicographic order (note that $n$ is the number of prime factors). Theorem 6.3 is proved by induction on this complexity. As the first step of the induction, we prove the following. 
Assertion 6.7. Suppose that $(n, p)=(1,1)$. Then Theorem 6.3 holds.

Proof of Assertion 6.7. Let $C_{1} \cup_{\Sigma} C_{2}$ be a minimal genus Heegaard splitting of $X^{(1)}$ and assume (without loss of generality) that $\partial X \subset \partial_{-} C_{1}$. Let $A$ be a Hopf spanning annulus.

Case 1. $C_{1} \cup_{\Sigma} C_{2}$ is strongly irreducible. In this case, we may suppose that each component of $A \cap \Sigma$ is a simple closed curve which is essential in $A$ and $\Sigma$. Minimize $|A \cap \Sigma|$ subject to this constraint (note that $A \cap \Sigma \neq \emptyset$ by (6) of Remarks 2.2). If $|A \cap \Sigma|=1$, then we have conclusion (1). Hence, suppose that $|A \cap \Sigma| \geq 2$. If some component of $A \cap C_{j}$ is compressible in $C_{j}$ (for $j=1$ or 2 ), then by compressing $A$ along that disk, we obtain a disk in $X$ with meridional boundary. This fact, together with the irreducibility of $X$, shows that $X$ is a solid torus, contradicting the assumption of Theorem 6.3 (note that $X_{1}=X$ ). Hence, every component of $A \cap C_{j}$ is incompressible in $C_{j}(j=1,2)$. This, together with the minimality of $|A \cap \Sigma|$, implies that each component of $A \cap C_{2}$ is essential in $C_{2}$. Hence, there is a meridian disk of $C_{2}$ which is disjoint from $A \cap C_{2}$ ((4) of Remarks 2.2). Then let $\mathcal{D}$ be a maximal system of mutually disjoint and non-parallel meridian disks of $C_{2}$ such that $\mathcal{D} \cap\left(A \cap C_{2}\right)=\emptyset$. Let $X^{\prime}$ be the manifold obtained by cutting $X^{(1)}$ along $A$, and let $\Sigma^{\prime}$ be the image of $\Sigma$ in $X^{\prime}$. Note that $X^{\prime}$ is homeomorphic to $X$. Let $\mathcal{S}^{\prime}$ be the surface obtained by compressing $\Sigma^{\prime}$ along (the image of) $\mathcal{D}$.

Claim 6.8. $\mathcal{S}^{\prime}$ is incompressible in $X^{\prime}$.

Proof. Suppose that there is a compressing disk $D$ for $\mathcal{S}^{\prime}$ in $X^{\prime}$. Regard $D$ as a disk in $X^{(1)}$. By the No Nesting Lemma [22], there exists a meridian disk $D^{\prime}$ in $C_{1}$ or $C_{2}$ with $\partial D^{\prime}=\partial D$. By using a standard innermost disk argument, we may suppose that $D^{\prime} \cap A=\emptyset$. Then, by isotopy, we may suppose that $D^{\prime} \cap \mathcal{D}=\emptyset$. By the maximality of $\mathcal{D}$, we see that $D^{\prime} \subset C_{1}$. Then, $D^{\prime} \cup \mathcal{D}$ provides a weak reduction for $\Sigma$, a contradiction.

If there is a component, say $S^{\prime}$, of $\mathcal{S}^{\prime}$ such that $\partial S^{\prime} \neq \emptyset$ and $S^{\prime}$ is not boundary parallel in $X^{\prime}$, then the corresponding surface in $X$ establishes conclusion (2) of Theorem 6.3 (note that $\chi\left(S^{\prime}\right) \geq 4-2 g$ ). Hence, we may suppose that each component of $\mathcal{S}^{\prime}$ is either closed or a boundary parallel annulus. Let $A_{1}$ and $A_{2}$ be the closures of the components of $A \backslash \Sigma$ such that $A_{1} \cap \partial X \neq \emptyset$ (hence $A_{1} \subset C_{1}$ ) and $A_{2}$ is adjacent to $A_{1}$ (i.e., $A_{2} \subset C_{2}$ and $\left.A_{2} \cap A_{1} \neq \emptyset\right)$; See figure 3 .

Let $C_{2}^{*}$ be the component of $\operatorname{cl}\left(C_{2} \backslash N(\mathcal{D})\right)$ which contains $A_{2}$. Note that $C_{2}^{*}$ naturally inherits a compression body structure from $C_{2}((2)$ of 


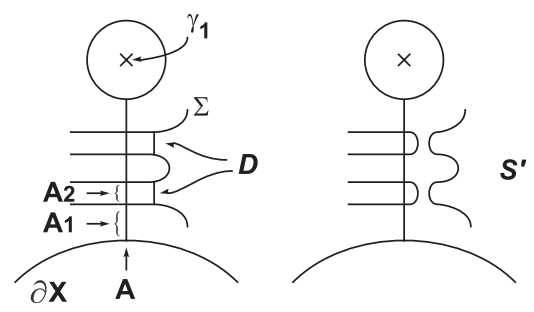

Figure 3: $\Sigma$ and $\mathcal{S}^{\prime}$.

Remarks 2.2). Since each component of $\mathcal{S}^{\prime}$ with non-empty boundary is an annulus, we see that $\partial_{+} C_{2}^{*}$ is a torus; hence either $C_{2}^{*}$ is a solid torus or it is a trivial compression body homeomorphic to (torus) $\times[0,1]$.

Subcase 1a. $\quad C_{2}^{*}$ is a solid torus; see figure 4 .

Let $A^{*}$ be an annulus obtained from $\partial C_{2}^{*}$ by performing surgery along $A_{1}$. We regard $A^{*}$ as an annulus properly embedded in $X\left(=X^{(1)} \cup N\left(\gamma_{1}\right)\right)$. If $A^{*}$ is not boundary parallel, then $K$ is not prime, contradicting our assumptions. Thus, $A^{*}$ is boundary parallel. If the boundary parallel region does not contain $C_{2}^{*}$, then $X$ is homeomorphic to the manifold obtained from $C_{2}^{*}$ by attaching the parallel region along an annulus corresponding to $A^{*}$. This shows that $X$ is a solid torus, contradicting the assumptions of Theorem 6.3. Hence the parallel region contains $C_{2}^{*}$. This implies that $A_{2}$ is a longitudinal annulus in $C_{2}^{*}$. Hence, the core curve of $A_{2}$, say $\gamma$, is a core curve of $C_{2}^{*}$. Since $\gamma$ is a core curve of $C_{2}^{*}$, we see that $\Sigma$ is a Heegaard surface of $\operatorname{cl}\left(X \backslash N\left(\gamma, C_{2}^{*}\right)\right)$. Then, $A_{1}$ and a part of $A_{2}$ form a Hopf spanning annulus for $\gamma$, and $\Sigma$ intersects this annulus in a single essential curve. Note that $\gamma_{1}$ is isotoped to $\gamma$ along $A$. Hence, $\operatorname{cl}\left(X \backslash N\left(\gamma, C_{2}^{*}\right)\right)$ is homeomorphic to $X^{(1)}$. These facts establish the conclusion (1) of Theorem 6.3.

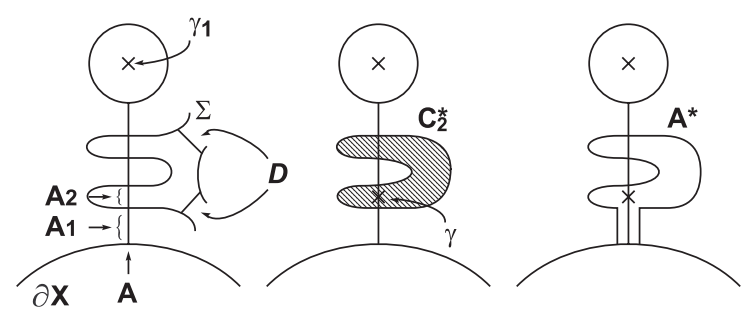

Figure 4: Subcase 1a. 


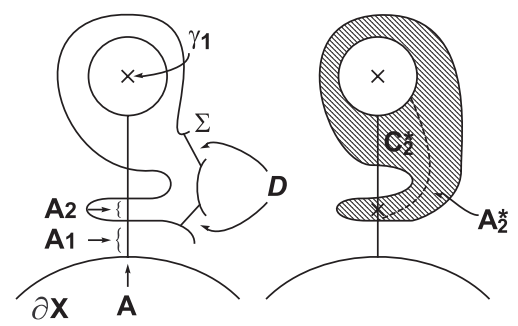

Figure 5: Subcase 1b.

Subcase 1b. $\quad C_{2}^{*}$ is a trivial compression body. Since $\partial X \subset C_{1}, \partial_{-} C_{2}^{*}=T_{1}$ (recall from Section 4 that $T_{1}=\partial N\left(\gamma_{1}\right)$ ). Note that there is a vertical annulus $A_{2}^{*}$ in $C_{2}^{*}$ such that $A_{2}^{*} \cap \partial_{+} C_{2}^{*}=A_{1} \cap \partial_{+} C_{2}^{*}$. Then, $A_{1} \cup A_{2}^{*}$ is a Hopf-Haken annulus for $\Sigma$ (figure 5), and we obtain conclusion (1) of Theorem 6.3.

Case 2. $C_{1} \cup_{\Sigma} C_{2}$ is weakly reducible. Let $\left(C_{1}^{1} \cup_{\Sigma^{1}} C_{2}^{1}\right) \cup \cdots \cup\left(C_{1}^{m} \cup_{\Sigma^{m}}\right.$ $C_{2}^{m}$ ) be a $\mathrm{S}-\mathrm{T}$ untelescoping of $C_{1} \cup_{\Sigma} C_{2}$ (Remark 2.5). Let $\mathcal{F}=\cup_{i=1}^{r} F_{i}$ be the collection of incompressible surfaces appearing in the untelescoping, where $F_{1}, \ldots, F_{r}$ are the connected components. Note that since $C_{1} \cup_{\Sigma} C_{2}$ is minimal genus, no $F_{i}$ is boundary parallel in $X^{(1)}$ (Remark 2.5). We may suppose that each component of $A \cap \mathcal{F}$ is a simple closed curve which is essential in $A$ and $\mathcal{F}$. Minimize $|A \cap \mathcal{F}|$ subject to this constraint.

Subcase 2a. $\quad \mathcal{F} \cap A \neq \emptyset$. Without loss of generality, we may suppose that $F_{1} \cap A \neq \emptyset$. Note that $\chi\left(F_{1}\right) \geq 6-2 g$. Let $X^{\prime}$ be the manifold obtained by cutting $X^{(1)}$ along $A$ (note that $X^{\prime} \cong X$ ) and $F^{\prime}$ the image of $F_{1}$ in $X^{\prime}$. Since each component of $F_{1} \cap A$ is essential in $F_{1}, F^{\prime}$ is incompressible in $X^{\prime}$. Hence, if there exists a component of $F^{\prime}$ which is not boundary parallel in $X^{\prime}$, then we have conclusion (2) of Theorem 6.3.

Thus, we may suppose that all components of $F^{\prime}$ are boundary parallel in $X^{\prime}$. In this case, each component of $F^{\prime}$ is an annulus. Let $A^{\prime}, A^{\prime \prime}$ be the images of $A$ in $\partial X^{\prime}$, and let $A_{X}^{\prime}, A_{T}^{\prime}$ be the images of $\partial X, T_{1}$ in $\partial X^{\prime}$, respectively. Note that $A_{X}^{\prime}, A_{T}^{\prime}$ are annuli such that $\partial X^{\prime}=A_{X}^{\prime} \cup A^{\prime} \cup A_{T}^{\prime} \cup$ $A^{\prime \prime}$; see figure 6 .

Suppose that there exists a component of $F^{\prime}$, say $A^{*}$, such that one component of $\partial A^{*}$ is contained in $A^{\prime}$ and the other in $A^{\prime \prime}$. Then $A^{*}$ is parallel to $A_{X}^{\prime}$ or $A_{T}^{\prime}$. We may suppose that $A^{*}$ is the outermost component with that property.

Suppose that the components of $\partial A^{*}$ are not identified in $F_{1}$ (see figure $7(\mathrm{~A})$ ). Let $A^{* *}$ be the component of $F^{\prime}$ which is adjacent to $A^{*}$ and 


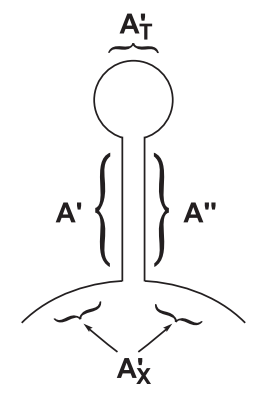

Figure 6: Subcase 2a.

is contained in the parallel region bounded by $A^{*}$. Then it is easy to see that $A^{* *}$ is parallel to an annulus in $A^{\prime}$ or $A^{\prime \prime}$. However, this contradicts the minimality of $|A \cap \mathcal{F}|$. Hence, the components of $\partial A^{*}$ are identified in $F_{1}$. This shows that $F_{1}$ is a boundary parallel torus in $X^{(1)}$, contradicting Remark 2.5.

Now suppose that for each component $A^{*}$ of $F^{\prime}$, we have that either $\partial A^{*} \subset A^{\prime}$ or $\partial A^{*} \subset A^{\prime \prime}$. Let us fix $A_{1}^{\prime}$, a component of $F^{\prime}$. We may suppose (without loss of generality) that $\partial A_{1}^{\prime} \subset A^{\prime}$. Let $A_{2}^{\prime}$ be another component of $F^{\prime}$ such that $A_{2}^{\prime}$ is adjacent to $A_{1}^{\prime}$ in $F_{1}$. Then $\partial A_{2}^{\prime} \subset A^{\prime \prime}$. Let $N_{1}^{\prime}$ be the parallel region between $A_{1}^{\prime}$ and a subsurface of $\partial X^{\prime}$. By the minimality of $|\mathcal{F} \cap A|$, we see that $N_{1}^{\prime} \cap \partial X^{\prime}$ is not contained in $A^{\prime}$. Hence, $N_{1}^{\prime} \cap \partial X^{\prime}$ contains $A^{\prime \prime}$, and $A_{2}^{\prime} \subset N_{1}^{\prime}$; see figure 7(B). However, this implies that $A_{2}^{\prime}$ admits a parallel region $N_{2}^{\prime}$ such that $N_{2}^{\prime} \cap \partial X^{\prime}$ is contained in $A^{\prime \prime}$, contradicting the minimality of $|\mathcal{F} \cap A|$. Hence, there exists a component of $F^{\prime}$ which is not boundary parallel, and this establishes conclusion (2) of Theorem 6.3.

Subcase 2b. $\mathcal{F} \cap A=\emptyset$. We may assume that $\partial X \subset C_{1}^{1}$. In this case, we basically apply the arguments of Case 1 of Assertion 6.7 to the strongly

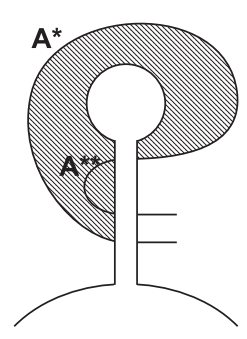

(A)

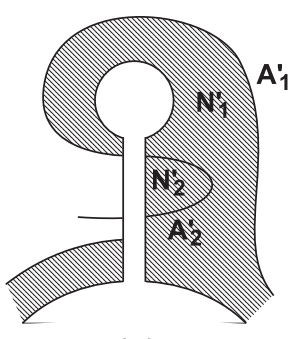

(B)

Figure 7: The annuli in Subcase 2a. 
irreducible Heegaard splitting $C_{1}^{1} \cup_{\Sigma^{1}} C_{2}^{1}$. Let $M=C_{1}^{1} \cup C_{2}^{1}$ (i.e., the ambient manifold of the Heegaard splitting $C_{1}^{1} \cup_{\Sigma^{1}} C_{2}^{1}$ ). Then, $A \subset M$. Since $C_{1}^{1} \cup_{\Sigma^{1}} C_{2}^{1}$ is strongly irreducible, we may suppose that each component of $A \cap \Sigma^{1}$ is a simple closed curve which is essential in both $A$ and $\Sigma^{1}$. Then by Remarks 2.2(6), $A \cap \Sigma^{1} \neq \emptyset$ and each component of $A \cap C_{i}^{1}$ is incompressible in $C_{i}^{1}(i=1,2)$. If $\left|A \cap \Sigma^{1}\right|=1$, then by Proposition 3.5 , we have conclusion (1) of Theorem 6.3. Hence, we may suppose that $\left|A \cap \Sigma^{1}\right| \geq 2$.

Let $A_{1}, A_{2}$ be the closures of the components of $A \backslash \Sigma^{1}$ such that $A_{1} \cap$ $\partial X \neq \emptyset$ (hence $A_{1} \subset C_{1}^{1}$ ) and $A_{2} \subset C_{2}^{1}$ with $A_{2} \cap A_{1} \neq \emptyset$. Then by arguments of Case 1 of Assertion 6.7, we can show that there exists a union of mutually disjoint meridian disks of $C_{2}^{1}$ (say $\mathcal{D}$ ) which is non-empty and $\mathcal{D} \cap\left(A \cap C_{2}^{1}\right)=\emptyset$. Let $M^{\prime}$ be the manifold obtained from $M$ by cutting along $A$, and let $\Sigma^{\prime}$ be the image of $\Sigma^{1}$ in $M^{\prime}$. Let $\mathcal{S}^{\prime}$ be the surface obtained by compressing $\Sigma^{\prime}$ along $\mathcal{D}$. Then by using the argument of the proof of Claim 6.8 in Case 1 of Assertion 6.7, we can show that $\mathcal{S}^{\prime}$ is incompressible in $M^{\prime}$.

If there is a component of $\mathcal{S}^{\prime}$ which is neither closed nor boundary parallel in $M^{\prime}$, then it is easy to see that the surface establishes conclusion (2) of Theorem 6.3. Therefore, we may suppose that each component of $\mathcal{S}^{\prime}$ is either closed or boundary parallel in $M^{\prime}$.

Let $C_{2}^{*}$ be the component of $\operatorname{cl}\left(C_{2}^{1} \backslash N(\mathcal{D})\right)$ which contains $A_{2}$. Then, $C_{2}^{*}$ is a compression body and $\partial_{+} C_{2}^{*}$ is a torus (for a detailed argument, see Case 1 of Assertion 6.7). Hence, we have the following three subcases:

Subcase A. $C_{2}^{*}$ is a solid torus,

Subcase B. $C_{2}^{*}$ is a trivial compression body homeomorphic to (torus) $\times[0,1]$ with $\partial_{-} C_{2}^{*}=T_{1}$, or

Subcase C. $C_{2}^{*}$ is a trivial compression body homeomorphic to (torus) $\times[0,1]$ with $\partial_{-} C_{2}^{*}=F_{k} \subset \mathcal{F}$.

For Subcases A and B, we apply the argument of Subcases 1a and 1b in Case 1 of Assertion 6.7 to show that there is a Hopf-Haken annulus for $\Sigma^{1}$ in $M$ with, possibly, the position of $\gamma_{1}$ changed. Then by Proposition 3.5, we have conclusion (1) of Theorem 6.3. We may therefore suppose that Subcase $\mathrm{C}$ holds; see figure 8.

Let $\widetilde{A}$ be the annulus obtained from $\partial_{+} C_{2}^{*}$ by performing surgery along $A_{1}$. Regard $\widetilde{A}$ as an annulus in $X\left(=X^{(1)} \cup N\left(\gamma_{1}\right)\right)$. If $\widetilde{A}$ is not boundary parallel, then we have a contradiction to $K$ being prime. Thus $\widetilde{A}$ is boundary parallel. Let $\widetilde{N}$ be the parallel region. Then, $\widetilde{N}$ is a solid torus and 


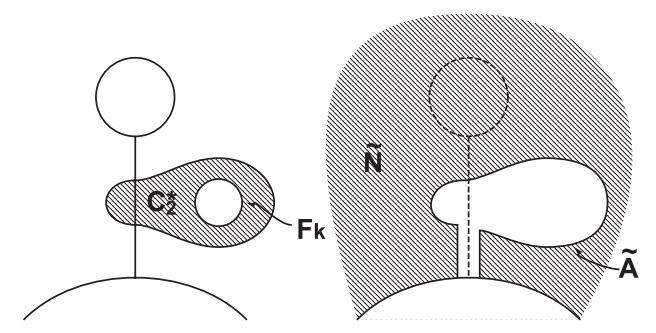

Figure 8: Subcase C.

$\widetilde{N} \cap \partial X=\partial \widetilde{N} \cap \partial X$ is a longitudinal annulus, that is, the annulus wraps around $\widetilde{N}$ once.

Suppose that $\tilde{N} \supset F_{k}$. Since $\partial_{+} C_{2}^{*}$ and $F_{k}$ are parallel, this implies that $F_{k}$ bounds a solid torus in $X$ which is isotopic to $\widetilde{N}$. Since $F_{k}$ is incompressible in $X^{(1)}, \gamma_{1}$ must be contained in this solid torus. Note that $A$ connects $\gamma_{1}$ to $\partial X$. However, this implies that $A \cap F_{k} \neq \emptyset$, contradicting the condition of Subcase 2b. Hence, we have $\widetilde{N} \cap F_{k}=\emptyset$ (see the right side of figure 8). This shows that $\partial_{+} C_{2}^{*}$ (hence also $F_{k}$ which is parallel to it) is boundary parallel in $X$. By Remark $2.5, F_{k}$ is not boundary parallel in $X^{(1)}$ and, therefore, $\gamma_{1}$ must be contained in the parallel region between $F_{k}$ and $\partial X$. Then recall that $A \cap F_{k}=\emptyset$ (the condition of Subcase 2b), and $\partial A$ consists of $\gamma_{1}$ and a meridian curve of $\partial X$. This shows that $M \cong D(2)$ (with $D(2) \cong\left(\right.$ disk with two holes) $\times S^{1}$, as defined in the end of Section 2 ).

As conclusions of the above, we have the following:

(1) $F_{k}$ is a boundary parallel torus in $X$, and

(2) $M$ is homeomorphic to a (disk with two holes) $\times S^{1}$, where $\partial M=$ $\partial X \cup T_{1} \cup F_{k}$.

Let $X^{*}=\operatorname{cl}\left(X^{(1)} \backslash M\right)(\cong X)$. Note that $M \cap X^{*}=F_{k}$. By Proposition 2.13 , there is a weakly reducing collection of disks $\Delta$ for $\Sigma$ such that $\widehat{\Sigma}(\Delta)=F_{k}$. Then by Proposition 2.9, we see that $g\left(X^{(1)}\right)=g(M)+$ $g\left(X^{*}\right)-1$. By $(2)$ of Remarks 3.2, there exists a minimal genus Heegaard splitting $\bar{C}_{1}^{\prime} \cup_{\bar{\Sigma}^{\prime}} \bar{C}_{2}^{\prime}$ of $M$ such that $\bar{\Sigma}^{\prime} \cap A$ consists of a single simple closed curve that is essential in $A$. Take the amalgamation of $\bar{C}_{1}^{\prime} \cup_{\bar{\Sigma}^{\prime}} \bar{C}_{2}^{\prime}$ and a minimal genus Heegaard splitting of $X^{*}$. Then by Lemma 2.7, and Proposition 3.5, we see that conclusion (1) of Theorem 6.3 holds.

This completes the proof of Assertion 6.7.

Assertion 6.9. Suppose that $n=1$. Then, Theorem 6.3 holds. 


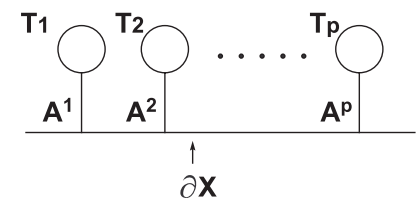

Figure 9: Hopf spanning annuli.

Proof of Assertion 6.9. Assertion 6.9 is proved by inducting on $p$. Assertion 6.7 gives the first step of the induction. Hence, we suppose that $p \geq 2$. Let $C_{1} \cup_{\Sigma^{(p)}} C_{2}$ be a minimal genus Heegaard splitting for $X^{(p)}$ and assume that $\partial X \subset \partial_{-} C_{1}$. Recall (from Section 4) that $\partial X^{(p)}=\partial X \cup T_{1} \cup$ $\cdots \cup T_{p}$. Let $A^{1}, \ldots, A^{p}$ be mutually disjoint Hopf spanning annuli between $\partial X$ and $T_{1}, \ldots, T_{p}$, respectively, and $A=\cup_{j=1}^{p} A^{j}$; see figure 9. By (6) of Remarks 2.2, we see that for each $j, A^{j} \cap \Sigma^{(p)} \neq \emptyset$.

Case 1. $C_{1} \cup_{\Sigma^{(p)}} C_{2}$ is strongly irreducible. In this case, we may suppose that $A \cap \Sigma^{(p)}$ consists of simple closed curves that are essential in $A$ and $\Sigma^{(p)}$. Subject to that constraint, we minimize $\left|A \cap \Sigma^{(p)}\right|$. If $\left|A^{j} \cap \Sigma^{(p)}\right|=1$ for some $j$, then we have conclusion (1) of Theorem 6.3. Hence, suppose that for each $j,\left|A^{j} \cap \Sigma^{(p)}\right| \geq 2$. The following argument is quite similar to that of Step 1 in the proof of Assertion 6.7; hence we will only sketch it here.

Let $\mathcal{D}$ be a maximal collection of meridian disks for $C_{2}$ so that $\mathcal{D} \cap(A \cap$ $\left.C_{2}\right)=\emptyset$; by (4) of Remarks $2.2, \mathcal{D} \neq \emptyset$. Let $X^{\prime}$ be the manifold obtained by cutting $X^{(p)}$ along $A$, and let $\Sigma^{\prime}$ be the image of $\Sigma^{(p)}$ in $X^{\prime}$. Let $\mathcal{S}^{\prime}$ be the surface obtained from $\Sigma^{\prime}$ by compressing along $\mathcal{D}$. Then by the same argument as in the proof of Claim 6.8 of the proof of Assertion 6.7, we see that $\mathcal{S}^{\prime}$ is incompressible in $X^{\prime}$.

If there exists a component of $\mathcal{S}^{\prime}$ with non-empty boundary that is not boundary parallel in $X^{\prime}$, then we have conclusion (2) of Theorem 6.3. Thus, we may assume that each component of $\mathcal{S}^{\prime}$ is either closed or boundary parallel. (Hence each component of $\mathcal{S}^{\prime}$ with non-empty boundary is a boundary parallel annulus.) Let $A_{1}, A_{2}$ be the closures of the components of $A^{1} \backslash \Sigma^{(p)}$ such that $A_{1} \cap \partial X \neq \emptyset$ (hence $A_{1} \subset C_{1}$ ) and that $A_{2} \subset C_{2}$ with $A_{2} \cap A_{1} \neq \emptyset$. Let $C_{2}^{*}$ be the component of $\operatorname{cl}\left(C_{2} \backslash N(\mathcal{D})\right)$ which contains $A_{2}$. Then $C_{2}^{*}$ is either a solid torus or homeomorphic to (torus) $\times[0,1]$.

Subcase 1a. $C_{2}^{*}$ is a solid torus. In this subcase, the argument of Subcase 1a of the proof of Assertion 6.7 can be applied to show that we can isotope $\gamma_{1}$ along $A^{1}$ to a core curve of $A_{2}$ to show that there exists a minimal genus Heegaard splitting of $X^{(p)}$ that intersects a Hopf spanning annulus in a single essential simple closed curve (conclusion (1) of Theorem 6.3). 
Subcase 1b. $C_{2}^{*}$ is homeomorphic to (torus) $\times[0,1]$. In this case, the argument of Subcase 1.b of the proof of Assertion 6.7 applies to show that conclusion (1) of Theorem 6.3 holds.

Case 2. $C_{1} \cup_{\Sigma^{(p)}} C_{2}$ is weakly reducible. Let

$$
\left(C_{1}^{1} \cup_{\Sigma^{1}} C_{2}^{1}\right) \cup \cdots \cup\left(C_{1}^{m} \cup_{\Sigma^{m}} C_{2}^{m}\right)
$$

be a S-T untelescoping of $C_{1} \cup_{\Sigma^{(p)}} C_{2}$, where $\partial X \subset \partial_{-} C_{1}^{1}$. Let $\mathcal{F}$ be the union of the essential surface appearing in the above untelescoping (Remark 2.5) and $\left\{F_{i}\right\}_{i=1}^{r}$ its connected components. We may suppose that each component of $\mathcal{F} \cap A$ is a simple closed curve that is essential in both $\mathcal{F}$ and $A$, and $|\mathcal{F} \cap A|$ is minimal subject to this constraint. Then we have the following subcases.

Subcase 2a. $\quad \mathcal{F} \cap A \neq \emptyset$. Without loss of generality, we may suppose that $F_{1} \cap A^{1} \neq \emptyset$. Let $F^{\prime}$ be the image of $F_{1}$ in $X^{\prime}\left(X^{\prime}\right.$ as in Case 1 of Assertion 6.9). Since each component of $F_{1} \cap A$ is essential in $F_{1}, F^{\prime}$ is incompressible in $X^{\prime}$. Hence, if there exists a component of $F^{\prime}$ which is not boundary parallel, then we have conclusion (2) of Theorem 6.3. We suppose that each component of $F^{\prime}$ is boundary parallel in $X^{\prime}$ (see figure 10).

This implies that $F^{\prime}$ can be isotoped to be contained in $N\left(\partial X^{\prime}, X^{\prime}\right)$. Here, we note that $X^{(p)}$ admits the decomposition

$$
X^{(p)}=X^{*} \cup_{T} D(p+1),
$$

where $X^{*} \cong X, D(p+1)$ is a (disk with $p+1$ holes) $\times S^{1}$ (see Section 2 ), and a meridian of $X^{*}$ is identified with $\{*\} \times S^{1}$, where $*$ is a point on the boundary of the disk.

Since $F^{\prime}$ is contained in $N\left(\partial X^{\prime}, X^{\prime}\right)$, we may suppose that $F_{1}$ is contained $\operatorname{in} \operatorname{int}(D(p+1))$. Then by $\left[6, \mathrm{VI} .34\right.$, VI.18], we see that $F_{1}$ is vertical, i.e., $F_{1}$ is isotopic to a torus of the form (simple closed curve) $\times S^{1}$ in $D(p+1)$.

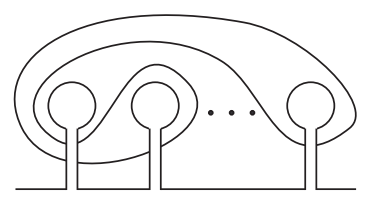

type 1

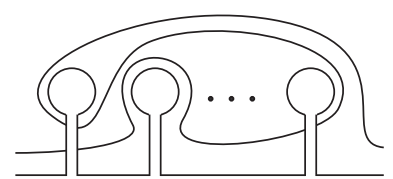

type 2

Figure 10: $F_{1}$ in Subcase 2a. 
Hence, $F_{1}$ gives the following decomposition:

$$
X^{(p)}=X^{\left(p_{1}\right)} \cup_{F_{1}} D\left(p_{2}\right),
$$

where $p_{1}+p_{2}=p+1$. We say that $F_{1}$ is of type 1 if $\partial X \subset \partial X^{\left(p_{1}\right)}$ and of type 2 if $\partial X \subset \partial D\left(p_{2}\right)$, see figure 10 .

Suppose that $F_{1}$ is of type 1 . Here, we note that $p_{2} \geq 2$ (Remark 2.5); hence $p_{1}<p$ and we may apply the inductive hypothesis on $X^{\left(p_{1}\right)}$, that is, there is a minimal genus Heegaard splitting $C_{1}^{\prime \prime} \cup_{\Sigma^{\prime \prime}} C_{2}^{\prime \prime}$ of $X^{\left(p_{1}\right)}$ and a Hopf spanning annulus $A_{1}^{\prime \prime} \subset X^{\left(p_{1}\right)}$ between $\partial X$ and $F_{1}$ so that $\Sigma^{\prime \prime} \cap A_{1}^{\prime \prime}$ is a single simple closed curve which is essential in $A_{1}^{\prime \prime}$. On the other hand, by (2) of Remarks 3.2, there is a minimal genus Heegaard splitting of $D\left(p_{2}\right)$ and an essential annulus $A_{2}^{\prime \prime} \subset D\left(p_{2}\right)$ such that the intersection of the Heegaard surface and $A_{2}^{\prime \prime}$ is a single simple closed curve which is essential in $A_{2}^{\prime \prime}$, and $A_{2}^{\prime \prime} \cap F_{1}=A_{1}^{\prime \prime} \cap F_{1}$. Then by applying Lemma 2.7 and Propositions 2.9 and 3.6 to these Heegaard splittings, we see that conclusion (1) of Theorem 6.3 holds.

Suppose that $F_{1}$ is of type 2. By Remark 2.5, we see that $\partial D\left(p_{2}\right)$ contains at least one $T_{i}$. By changing subscripts if necessary, we may suppose that $T_{1} \subset \partial D\left(p_{2}\right)$. We take a minimal genus Heegaard splitting of $D\left(p_{2}\right)$ and an essential annulus $A_{2}^{\prime \prime} \subset D\left(p_{2}\right)$ such that $A_{2}^{\prime \prime}$ intersects the Heegaard surface in a single simple closed curve which is essential in $A_{2}^{\prime \prime}$ and that $A_{2}^{\prime \prime}$ connects $\partial X$ to $T_{1}$ (Remarks 3.2(2)). Then, take an amalgamation of this Heegaard splitting and a minimal genus Heegaard splitting of $X^{\left(p_{1}\right)}$. By Lemma 2.7 and Propositions 2.9 and 3.5, we see that we have conclusion (1) of Theorem 6.3.

Subcase 2b. $\mathcal{F} \cap A=\emptyset$. We apply arguments similar to that of Case 1 of Assertion 6.9 to the strongly irreducible Heegaard splitting $C_{1}^{1} \cup_{\Sigma^{1}} C_{2}^{1}$. Let $M=C_{1}^{1} \cup C_{2}^{1}$. By the argument of Case 1 of Assertion 6.9 of the proof of Assertion 6.7, we see that after isotopy each component $A \cap C_{2}^{1}$ is essential in $C_{2}^{1}$. Let $\mathcal{D}$ be a maximal collection of meridian disks for $C_{2}^{1}$ such that $\mathcal{D} \cap\left(A \cap C_{2}^{1}\right)=\emptyset$ (as above, $\mathcal{D} \neq \emptyset$ ). Let $M^{\prime}$ be the manifold obtained by cutting $M$ along $A$, and let $\Sigma^{\prime}$ be the image of $\Sigma^{1}$ in $M^{\prime}$. Let $\mathcal{S}^{\prime}$ be the surface obtained by compressing $\Sigma^{\prime}$ along $\mathcal{D}$. Then by using the argument of Claim 6.8 in the proof of Assertion 6.7, we can show that $\mathcal{S}^{\prime}$ is incompressible in $M^{\prime}$.

If there is a component of $\mathcal{S}^{\prime}$ with non-empty boundary that is not boundary parallel, then we have conclusion (2) of Theorem 6.3. Suppose then that each component of $\mathcal{S}^{\prime}$ is either closed or boundary parallel in $X^{\prime}$. As before, let $A_{1}, A_{2}$ be the closures of components of $A^{1} \backslash \Sigma^{1}$ such that 
$A_{1} \cap \partial X \neq \emptyset$ and that $A_{2} \subset C_{2}^{1}$ with $A_{2} \cap A_{1} \neq \emptyset$. Let $C_{2}^{*}$ be the component of $\operatorname{cl}\left(C_{2}^{1} \backslash N(\mathcal{D})\right)$ containing $A_{2}$. As in the proof of Assertion 6.7, we have the following subcases.

Subcase A. $\quad C_{2}^{*}$ is a solid torus.

Subcase B. $C_{2}^{*}$ is a trivial compression body homeomorphic to (torus) $\times[0,1]$ with $\partial_{-} C_{2}^{*}=T_{i}$.

Subcase C. $C_{2}^{*}$ is a trivial compression body homeomorphic to (torus) $\times[0,1]$ with $\partial_{-} C_{2}^{*}=F_{k} \subset \mathcal{F}$.

For Subcases A and B, we apply the arguments of Subcases 1a and 1b in Case 1 of Assertion 6.9 and using Proposition 3.5 conclude that we have conclusion (1) of Theorem 6.3.

Suppose Subcase $\mathrm{C}$ holds (figure 8 ). Let $\widetilde{A}$ be an annulus obtained from $\partial_{+} C_{2}^{*}$ by performing surgery along $A_{1}$. Regard $\widetilde{A}$ as an annulus in $X\left(=X^{(p)} \cup\left(\cup_{i=1}^{p} N\left(\gamma_{i}\right)\right)\right)$. If $\widetilde{A}$ is not boundary parallel, then we have a contradiction to our assumption that $n=1$. Therefore, we may suppose that $\widetilde{A}$ is boundary parallel in $X$. Then by using the argument of Subcase C of Subcase $2 \mathrm{~b}$ of the proof of Assertion 6.7, we see that the following hold. (Note that $A \subset M \cup\left(\cup_{i=1}^{p} N\left(\gamma_{i}\right)\right)$.)

(1) $F_{k}$ is boundary parallel in $X$, and

(2) $M$ is homeomorphic to $D(p+1)$, where $\partial M=\partial X \cup F_{k} \cup\left(T_{1} \cup \cdots \cup\right.$ $\left.T_{p}\right)$.

This gives the following decomposition:

$$
X^{(p)}=X \cup_{F_{k}} D(p+1) .
$$

Then by the inductive hypothesis, Lemma 2.7 and Remark 3.2 Propositions 2.9, and 3.5, we see that conclusion (1) of Theorem 6.3 holds (for detailed arguments, see the last paragraph of Subcase 2a of this proof).

This completes the proof of Assertion 6.9.

We now complete the proof of Theorem 6.3. Recall that the proof is carried out by induction on $(n, p)$ with the lexicographic ordering. By Assertion 6.9, we may assume that $n \geq 2$. Let $C_{1} \cup_{\Sigma} C_{2}$ be a minimal genus Heegaard splitting of $X^{(p)}$. By Theorem 4.1, we either have conclusion (2) of Theorem 6.3, or $C_{1} \cup_{\Sigma} C_{2}$ weakly reduces to a swallow follow torus $T$, 
which gives the following decomposition of $X^{(p)}$ :

$$
X^{(p)}=\mathcal{X}_{1}^{\left(p_{1}+1\right)} \cup_{T} \mathcal{X}_{2}^{\left(p_{2}\right)},
$$

where $\partial X \subset \partial \mathcal{X}_{1}^{\left(p_{1}+1\right)}, p_{1}+p_{2}=p$ and $\mathcal{X}_{1}=E\left(\mathcal{K}_{1}\right), \mathcal{X}_{2}=E\left(\mathcal{K}_{2}\right)$ (possibly $\mathcal{K}_{1}$ or $\mathcal{K}_{2}$ being the trivial knot in $S^{3}$ ) and $K=\mathcal{K}_{1} \# \mathcal{K}_{2}$.

Case 1. $\mathcal{K}_{1}$ is the trivial knot in $S^{3}$. In this case, $\mathcal{X}_{1}^{\left(p_{1}+1\right)}$ is (disk with $p_{1}+1$ holes) $\times S^{1}, p_{1} \geq 1$ (Remark 2.5). By changing the subscripts if necessary, we may suppose that $T_{1} \subset \partial \mathcal{X}_{1}^{\left(p_{1}+1\right)}$. By (2) of Remarks 3.2, there exists a minimal genus Heegaard splitting $C_{1}^{1} \cup_{\Sigma^{1}} C_{2}^{1}$ of $\mathcal{X}_{1}^{\left(p_{1}+1\right)}$ and an essential annulus $A$ in $\mathcal{X}_{1}^{\left(p_{1}+1\right)}$ such that $A$ connects $\partial X$ to $T_{1}, A \cap \partial X$ is a meridian and $\Sigma^{1} \cap A$ is a single simple closed curve that is essential in $A$. Then by Lemma 2.7 and Propositions 2.9 and 3.5, we see that conclusion (1) of Theorem 6.3 holds.

Case 2. $\mathcal{K}_{2}$ is the trivial knot in $S^{3}$. In this case, $\mathcal{X}_{2}^{\left(p_{2}\right)}$ is (disk with $p_{2}$ holes) $\times S^{1}, p_{2} \geq 2$ (Remark 2.5). By changing the subscripts if necessary, we may suppose that $T_{1} \subset \partial \mathcal{X}_{2}^{\left(p_{2}\right)}$. Since $p_{1} \geq 2$, we have that $p_{1} \leq p-1$, and this allows us to apply the inductive hypothesis to $\mathcal{X}_{1}^{\left(p_{1}+1\right)}$. If conclusion (2) holds for $\mathcal{X}_{1}^{\left(p_{1}+1\right)}$, then conclusion (2) holds for $X^{(p)}$ (note that $K=\mathcal{K}_{1}$, and $\left.g\left(\mathcal{X}_{1}^{\left(p_{1}+1\right)}\right) \leq g\left(X^{(p)}\right)\right)$. Hence we may suppose that conclusion (1) holds for $\mathcal{X}_{1}^{\left(p_{1}+1\right)}$, i.e., there exists a minimal genus Heegaard splitting (say $C_{1}^{1} \cup_{\Sigma^{1}} C_{2}^{1}$ ) of $\mathcal{X}_{1}^{\left(p_{1}+1\right)}$ admitting a Hopf-Haken annulus $A^{*}$ so that $A^{*} \cap T \neq \emptyset$. By (2) of Remarks 3.2, there exists a minimal genus Heegaard splitting $C_{1}^{2} \cup_{\Sigma^{2}} C_{2}^{2}$ of $\mathcal{X}_{2}^{\left(p_{2}\right)}$ and an essential annulus $A$ in $\mathcal{X}_{2}^{\left(p_{2}\right)}$ such that $A$ connects $T_{1}$ to $T$, $A \cap T=A^{*} \cap T$ and $A \cap \Sigma^{2}$ is a simple closed curve which is essential in $A$. Then by Lemma 2.7 and Propositions 2.9 and 3.6, we see that $C_{1}^{1} \cup_{\Sigma^{1}} C_{2}^{1}$ and $C_{1}^{2} \cup_{\Sigma^{2}} C_{2}^{2}$ are amalgamated to give a minimal genus Heegaard splitting of $X^{(p)}$ admitting a Hopf-Haken annulus; hence conclusion (1) of Theorem 6.3 holds.

Case 3. Both $\mathcal{K}_{1}$ and $\mathcal{K}_{2}$ are non-trivial knots. Then we have the following subcases:

Subcase 3a. $p_{2}=0$. Since $p_{1}+p_{2}=p>0$, this implies that $p_{1}>0$. Note that $T_{1}$ is contained in $\mathcal{X}_{1}^{\left(p_{1}+1\right)}$. Recall that we used lexicographic order on $(n, p)$ and since $\mathcal{K}_{2}$ is a non-trivial knot, the number of prime factors of $\mathcal{K}_{1}$ is strictly less than that of $K$. We may therefore apply the inductive 
hypothesis to $\mathcal{X}_{1}^{\left(p_{1}+1\right)}$. If conclusion (2) of Theorem 6.3 holds for $\mathcal{X}_{1}^{\left(p_{1}+1\right)}$, then conclusion (2) holds for $X^{(p)}$. Suppose that conclusion (1) holds for $\mathcal{X}_{1}^{\left(p_{1}+1\right)}$. Then we obtain a minimal genus Heegaard splitting $C_{1}^{1} \cup_{\Sigma^{1}} C_{2}^{1}$ of $\mathcal{X}_{1}^{\left(p_{1}+1\right)}$ and an essential annulus $A$ connecting $\partial X$ to $T_{1}$ such that $A \cap \Sigma^{1}$ is a single simple closed curve which is essential in $A$. Then by Lemma 2.7 and Propositions 2.9 and 3.5, we see that conclusion (1) of Theorem 6.3 holds.

Subcase 3b. $p_{2}>0$. We may assume that $T_{1}$ is contained in $\mathcal{X}_{2}^{\left(p_{2}\right)}$. Apply the inductive hypothesis to both $\mathcal{X}_{1}^{\left(p_{1}+1\right)}$ and $\mathcal{X}_{2}^{\left(p_{2}\right)}$. If conclusion (2) of Theorem 6.3 holds for $\mathcal{X}_{1}^{\left(p_{1}+1\right)}$ or $\mathcal{X}_{2}^{\left(p_{2}\right)}$, then conclusion (2) holds for $X^{(p)}$. Thus, we may assume that conclusion (1) holds for both $\mathcal{X}_{1}^{\left(p_{1}+1\right)}$ and $\mathcal{X}_{2}^{\left(p_{2}\right)}$. Then we obtain minimal genus Heegaard splittings $C_{1}^{1} \cup_{\Sigma^{1}} C_{2}^{1}$ and $C_{1}^{2} \cup_{\Sigma^{2}}$ $C_{2}^{2}$, respectively, and Hopf-Haken annuli $A_{1} \subset \mathcal{X}_{1}^{\left(p_{1}+1\right)}$ and $A_{2} \subset \mathcal{X}_{2}^{\left(p_{2}\right)}$ for $\Sigma^{1}$ and $\Sigma^{2}$, with $A_{1}$ connecting $\partial X$ to $T$ and $A_{2}$ connecting $T$ to $T_{1}$, and $A_{1} \cap T=A_{2} \cap T$. Then by Lemma 2.7 and Propositions 2.9 and 3.6, we see that conclusion (1) of Theorem 6.3 holds.

This completes the proof of Theorem 6.3.

\section{Morimoto's conjecture for m-small knots}

Proof of Theorem 1.4. For the "if" part of Theorem 1.4, recall that $g\left(E\left(\#_{i \in I} K_{i}\right)\right) \leq \Sigma_{i \in I} g\left(E\left(K_{i}\right)\right)$ and $g\left(E\left(\#_{i \notin I} K_{i}\right)\right) \leq \Sigma_{i \notin I} g\left(E\left(K_{i}\right)\right)$ hold in general. If $E\left(\#_{i \in I} K_{i}\right)$ admits a primitive meridian, then by Proposition 1.2 we have that $g(X)<g\left(E\left(\#_{i \in I} K_{i}\right)\right)+g\left(E\left(\#_{i \notin I} K_{i}\right)\right)$. Combining these inequalities shows that $g(X)<\sum_{i=1}^{n} g\left(E\left(K_{i}\right)\right)$ as required.

Assume for a contradiction that the "only if" part of Theorem 1.4 is false and let $\left\{K_{i} \mid i=1, \ldots, n\right\}$ be a collection of $\mathrm{m}$-small knots that gives a counterexample, which minimizes $n$ among all such counterexamples. (Note that we may assume the uniqueness of prime decomposition of $\#_{i=1}^{n} K_{i}$ by Claim 4.4 of the proof of Theorem 4.1.) Let $X_{i}=E\left(K_{i}\right)$ and $X=E\left(\#_{i=1}^{n} K_{i}\right)$; this and all other notation is kept as in the previous sections. Let $\Sigma \subset X$ be a minimal genus Heegaard surface. By Corollary $4.3, \Sigma$ weakly reduces to a swallow follow torus, say $T$, giving the decomposition:

$$
X=X_{I}^{(1)} \cup_{T} X_{J}
$$

where $I \cup J=\{1, \ldots, n\}, I \neq \emptyset, J \neq \emptyset, I \cap J=\emptyset, X_{I}=E\left(\#_{i \in I} K_{i}\right)$ and $X_{J}=E\left(\#_{i \in J} K_{i}\right)$.

By Corollary 5.4, we know that either $g\left(X_{I}\right)=g\left(X_{I}^{(1)}\right)$ or $g\left(X_{I}\right)=$ $g\left(X_{I}^{(1)}\right)-1$. If $g\left(X_{I}\right)=g\left(X_{I}^{(1)}\right)$, then by Corollary $6.5, X_{I}$ admits a 
primitive meridian and we have a contradiction to our assumption that $\left\{K_{i}\right\}$ forms a counterexample. We may therefore assume that $g\left(X_{I}^{(1)}\right)-1=$ $g\left(X_{I}\right)$. By Corollary 5.4, $g(X)=g\left(X_{I}\right)+g\left(X_{J}\right)$. Therefore, by assumption, we have that $g\left(X_{I}\right)+g\left(X_{J}\right)=g(X)<\Sigma_{i=1}^{n} g\left(X_{i}\right)=\Sigma_{i \in I} g\left(X_{i}\right)+\Sigma_{i \in J}$ $g\left(X_{i}\right)$. Therefore, either $g\left(X_{I}\right)<\Sigma_{i \in I} g\left(X_{i}\right)$ or $g\left(X_{J}\right)<\Sigma_{i \in J} g\left(X_{i}\right)$ must hold (say the former); by the assumption of minimality on $n, X_{I}$ cannot give a counterexample; therefore for some $\emptyset \neq I^{\prime} \subset I$, the exterior of $\#_{i \in I^{\prime}} K_{i}$ admits a primitive meridian, contradicting the assumption that the collection $\left\{K_{i} \mid i=1, \ldots, n\right\}$ provides a counterexample.

This completes the proof of Theorem 1.4.

\section{Acknowledgments}

We would like to thank Kanji Morimoto for helpful conversations and the referees for many helpful comments. We thank Sean Bowman for a careful reading of an early manuscript. The second named author: this research started while I was a JSPS fellow at Nara Women's University and completed while I was a member of 21st-century COE program "Constitution for wide-angle mathematical basis focused on knots" at Osaka City University, leader: Akio Kawauchi. I would like to thank both universities, the Math departments, Tsuyoshi Kobayashi and Akio Kawauchi for wonderfully warm hospitality. T.K. was supported by Grant-in-Aid for scientific research, JSPS grant number 00186751. Y.R. was supported in part by JSPS (fellow number P00024) and by the 21st century COE program "Constitution for wide-angle mathematical basis focused on knots" (Osaka City University); leader: Akio Kawauchi.

\section{References}

[1] M. Boileau and J.-P. Otal, Sur les scindements de Heegaard du tore $T^{3}$, J. Diff. Geom. 32 (1990), no. 1, 209-233.

[2] A.J. Casson and C. McA. Gordon, Reducing Heegaard splittings, Topol. Appl., 27 (1987), no. 3, 275-283.

[3] W. Haken, Some results on surfaces in 3-manifolds. Studies in Modern Topology, Math. Assoc. Amer. (distributed by Prentice-Hall, Englewood Cliffs, NJ), 1968, 39-98.

[4] A. Hatcher and W. Thurston, Incompressible surfaces in 2-bridge knot complements. Invent. Math. 79 (1985), no. 2, 225-246. 
[5] J. Hempel, 3-Manifolds, Princeton University Press, Princeton, NJ, 1976; Ann. Math. Stud. 86 (1976).

[6] W. Jaco, Lectures on three-manifold topology, CBMS Regional Conference Series in Mathematics, vol. 43, American Mathematical Society, Providence, RI, 1980.

[7] T. Kobayashi, A construction of arbitrarily high degeneration of tunnel numbers of knots under connected sum, J. Knot Theory Ramifi. 3 (1994), no. 3, 179-186.

[8] T. Kobayashi, Heegaard splittings of exteriors of two bridge knots, Geom. Topol. 5 (2001), 609-650 (electronic).

[9] T. Kobayashi and Y. Rieck, Local detection of strongly irreducible Heegaard splittings via knot exteriors, Topol. Appl. 138 (2004), nos. 1-3, 239-251.

[10] T. Kobayashi and Y. Rieck, On the growth rate of tunnel number of knots, J. Reine Angew. Math. 592 (2006), 63-78.

[11] K. Miyazaki, Conjugation and the prime decomposition of knots in closed, oriented 3-manifolds. Trans. Amer. Math. Soc. 313 (1989), no. $2,785-804$.

[12] Y. Moriah, On boundary primitive manifolds and a theorem of CassonGordon, Topol. Appl. 125 (2004), no. 3, 571-579.

[13] Y. Moriah and H. Rubinstein, Heegaard structures of negatively curved 3-manifolds, Comm. Anal. Geom. 5 (1997), no. 3, 375-412.

[14] K. Morimoto, On composite tunnel number one links, Topol. Appl. 59 (1994), 59-71.

[15] K. Morimoto, There are knots whose tunnel numbers go down under connected sum, Proc. Amer. Math. Soc. 123 (1995), no. 11, 3527-3532.

[16] K. Morimoto, On the super additivity of tunnel number of knots, Math. Ann. 317 (2000), no. 3, 489-508.

[17] K. Morimoto, Tunnel number, connected sum and meridional essential surfaces, Topology 39 (2000), no. 3, 469-485.

[18] K. Morimoto, M. Sakuma and Y. Yokota, Examples of tunnel number one knots which have the property " $1+1=3$ ", Math. Proc. Cambridge Philos. Soc. 119 (1996), no. 1, 113-118. 
[19] Y. Rieck, Heegaard structures of manifolds in the Dehn filling space, Topology 39 (2000), no. 3, 619-641.

[20] Y. Rieck and E. Sedgwick, Finiteness results for Heegaard surfaces in surgered manifolds, Comm. Anal. Geom. 9 (2001) no. 2, 351-367.

[21] Y. Rieck and E. Sedgwick, Persistence of Heegaard structures under Dehn filling, Topol. Appl. 109 (2001), no. 1, 41-53.

[22] M. Scharlemann, Local detection of strongly irreducible Heegaard splittings, Topol. Appl. 90 (1998), no. 1-3, 135-147.

[23] M. Scharlemann, Heegaard splittings of compact 3-manifolds, Handbook of Geometric Topology, North-Holland, Amsterdam, 2002, 921-953.

[24] M. Scharlemann and A. Thompson, Thin position for 3-manifolds, Geometric topology (Haifa, 1992), vol. 164, Contemp. Math., American Mathematical Society, Providence, RI, 1994, 231-238.

[25] J. Schultens, The classification of Heegaard splittings for (compact orientable surface) $\times S^{1}$, Proc. London Math. Soc. (3) 67 (1993), no. 2, 425-448.

[26] J. Schultens, Heegaard splittings of Seifert fibered spaces with boundary, Trans. Amer. Math. Soc. 347 (1995), 2533-2552.

[27] E. Sedgwick, Genus two 3-manifolds are built from handle number one pieces, Algebr. Geom. Topol. 1 (2001), 763-790 (electronic).

[28] F. Waldhausen, Heegaard-Zerlegungen der 3-Sphäre, Topology 7 (1968), 195-203.

DEPARTMENT OF MATHEMATICS

NARA WOMEN's University KitaUoya NishimaChI

NARA 630-8506

JAPAN

E-mail address: tsuyoshi@cc.nara-wu.ac.jp

DEPARTMENT OF MATHEMATICAL SCIENCES

UNIVERSITY OF ARKANSAS

FAYETTEVILLE

AR 72701, USA

E-mail address: yoav@uark.edu

Received March 11, 2005 
